\title{
European Competition Policy Modernization: From Notifications to Legal Exception*
}

\author{
Frédéric Loss ${ }^{\dagger} \quad$ Estelle Malavolti-Grimal ${ }^{\ddagger} \quad$ Thibaud Vergé ${ }^{\S}$ \\ Fabian Bergès-Sennou
}

January 2006

\begin{abstract}
Council Regulation (EC) 1/2003 came into force on the $1^{\text {st }}$ of May 2004 replacing the mandatory notification of agreements between firms by a regime of ex post monitoring. This paper provides a theoretical justification for this shift based on the competition authority's accuracy of judgement. We show that ex post monitoring dominates when the competition authority's probability of error if low enough. We also investigate the two other existing legal systems, i.e. block exemptions and black list, and show that they should be preferred when the competition authority's beliefs about the welfare impact of the agreements are very optimistic or very pessimistic.
\end{abstract}

Keywords: competition policy, imperfect audit.

JEL classification: L4, D8.

${ }^{*}$ We would like to thank David Alary, Eric Avenel, Pedro Pita Barros, Bruno Jullien, Denis Gromb, Jean Marie Lozachmeur, Massimo Motta, Patrick Rey, Karl Schlag, Jean Tirole, the editor and two anonymous referees for helpful comments. Frédéric Loss, Estelle Malavolti-Grimal, and Thibaud Vergé gratefully acknowledge financial support from the European Commission (TMR while at FMG-LSE), the European University Institute and the Leverhulme Trust (while at CMPO, Bristol) respectively.

${ }^{\dagger}$ CNAM - Laboratoire d'économétrie.

‡LEEA-ENAC (Toulouse).

$\S$ Laboratoire d'Economie Industrielle, CREST-INSEE.

ฯUniversity of Toulouse (INRA-ESR). 


\section{Introduction}

In January 2003, the European Commission (henceforth EC) published a new regulation modifying the procedure for the detection of anti-competitive practices and abuses of dominant position. ${ }^{1}$ The main effects of this reform are to replace the current notification system of ex ante monitoring in favor of an ex post offence repression regime and to decentralize the enforcement of competition rules by strengthening the Member States' authorities powers and jurisdiction.

The European competition policy towards inter-firms agreements relies mostly on article 81 of the European Community Treaty. Under article 81(1) "shall be prohibited (...) any agreement (...) which may affect trade between Member States and which have as their object or effect the prevention, restriction or distortion of competition within the common market." Price-fixing or market-sharing agreements (such as resale price maintenance for instance) belong to this category. However, under article 81(3) some agreements can nevertheless be declared valid if they promote economic progress or enhance the distribution of goods within the Common Market and consumers get a "fair share of the resulting benefit." For instance, R\&D joint ventures or even some exclusive distribution agreements might satisfy these conditions.

In 1962, regulation 17 setting the rules for the implementation of article 81 was published by the EC and stated that individual exemption (under article 81(3)) could only be granted if the agreement had been notified to the EC. The EC decided at the time to keep the exclusive right to grant exemption in order to develop a coherent law enforcement and to diffuse a competition culture within each of the member states. A broad interpretation of the concept of "restriction to competition" combined to the fact that regulation $17 \mathrm{im}$ posed a response for each notification led to the EC being quickly overwhelmed: ${ }^{2}$ by 1967 more than 37450 cases were pending. The EC thus took several steps in order to limit the number of notifications and speed up the process: "general notices" were published, the concept of "appreciable effect on competition" was introduced to ignore minor cases and the block exemptions regulations were set up. At the same time, some restrictive clauses (black list) were deemed anticompetitive and disqualified the agreements from qualifying under block exemptions system. Finally, the EC extensively started to close

\footnotetext{
${ }^{1}$ The new regulation was adopted in the context of the modernization of European competition law and replaces regulation 17/1962 as from the 1st May 2004. See Council Regulation (EC) 1/2003, Official Journal of the European Communities, $4^{\text {th }}$ January 2003.

${ }^{2}$ See Verouden (2003) who provides a detailed analysis of the evolution of the EC interpretation of article 81.
} 
cases informally ("comfort letters"). Nevertheless, problems still remained: less than 20 formal decisions were taken every year in the early 1990's, although the annual number of notified cases had been reduced to around 250. Moreover, less than $1 \%$ of the notifications led to a prohibition decision, suggesting that some resources could have been saved. This pushed the EC to reform the system, trying to refocus antitrust intervention on the most severe anti-competitive practices such as cartels. In order to free the much needed resources, the EC decided to decentralize the enforcement of competition rules to the Member States and to remove the costly and ineffective notification system. The new legal exception regime came into force on $1^{\text {st }}$ May 2004.

This paper provides a framework to compare the efficiency of these different systems: notifications, legal exception, block exemptions and black list. We claim that the shift from the notification regime to the legal exception regime is relevant only if the quality of judgement of the EC is sufficiently high. We show that if the accuracy of the evaluation achieved during the investigation is high enough, it is possible to deter the signature of some harmful agreements only, by setting the appropriate fines in the ex post regime. If the quality of evaluation is low, fines would deter the signature of welfare improving agreements as well. In this case, early intervention is preferable and a notification system can thus be more effective than a legal exception regime. In other words, a higher quality of evaluation by the Competition Authority (henceforth $C A$ ) advocates in favor of the $e x$ post control regime. Forty years of experience have given the EC a better knowledge of the market organization, of the competition mechanisms and of the impact of agreements on economic welfare. The EC evaluations are therefore likely to be better now, which may justify the change towards an ex post control of the agreements. However, this may not have been the case in 1962 explaining the initial preference for a notification regime. Besides, the relative costs of type I and type II errors are also important factors in the choice of regime: we show that when the accuracy of evaluation is low and type II errors might be very costly, the black list system banning any agreement is preferred, while block exemptions regulations can be justified whenever type I errors become too costly relative to type II errors.

In contrast to the literature on regulation, few theoretical papers have focused on optimal competition policy design and implementation problems. ${ }^{3}$ The recent debate

\footnotetext{
${ }^{3}$ Rey (2003) provides a detailed presentation of competition policy design and implementation issues, and proposes a broad review of the existing literature. Cartel prosecution has the major area of interest for economic theory with a wide literature following works by Baron and Besanko (1984), Reinganum and Wilde (1985b) and Besanko and Spulber (1989). In the recent years, cartel prosecution has again
} 
about the reform of European competition policy has however triggered some research on the subject.

First, this reform is going to be welfare improving only if the national courts maintain the necessary unity in the EC competition rules enforcement. Mavroidis and Neven (2000, 2001) point out that simultaneous enforcement by several authorities is likely to occur and that each Member State will have little incentives to take into account the interests of other Member States. They show that decentralization may have a disintegrating effect and advocate for the emergence a formal coordination procedure.

Second, to be welfare improving, the legal exception regime has to be at least as efficient as the notification regime. Barros (2003) and Neven (2001) focus on the impact that the proposed change might have on the type of agreements that firms might implement and explicitly model the firms' reactions to the different policies. Barros (2003) considers identical firms and focuses on the impact of the reduced legal security created by the removal of the notification regime. He shows that the proposed reform may have two opposite effects: on the one hand, the probability of audit being reduced in the $e x$ post regime, firms might be tempted to sign more restrictive agreements. On the other hand, as legal uncertainty increases, they are likely to take less risk. The latter effect is however more likely to prevail, implying that the reform should result in firms signing less restrictive agreements. Neven (2001) considers heterogenous firms and focuses on the decision to notify potential agreements. He shows that the distribution of agreements implemented by firms should be more dispersed. Ex post monitoring indeed leads to a higher transaction cost thereby leading firms to sign agreements that are more likely to be cleared by the competition authority. Some beneficial agreements are therefore no longer implemented. Moreover, removing the notification regime induces some anti-competitive agreements which would have been notified otherwise to be implemented and not monitored. This advocates for a rather high level of ex post audit to limit the impact of these errors.

Although closely related to these two papers, our work differs in significant ways. In their papers, firms are uncertain on whether an agreement is harmful or not and the audit is used to elicit this information. We assume instead that firms are aware of the status of the agreement and analyze the impact of a given policy on the firms decision to sign an agreement. Considering a model of imperfect audit, we then compare the ex post and ex ante regimes, looking as well as per se rules such as black list or block exemptions been given attention following the implementation of leniency programs in the US and in Europe (See for instance Motta and Polo (2003) or Spagnolo (2000)). 
regulations.

The paper is organized as follows. The next section presents the framework and briefly describes the different legal systems. We first analyse the legal exception regime in section 3 and then compare it with a mixed regime involving notifications (section 4). Section 5 discusses these results and concludes. Most proofs are relegated in Appendices.

\section{The Model}

\subsection{The Firms and the Competition Authority}

There exists a continuum of size 1 of pairs of firms that can enter into agreements or decisions which may be judged as harmful for social welfare by a Competition Authority. ${ }^{4}$ Pairs of firms are divided up into two groups:

- A first category of pairs of firms have the opportunity to sign a "bad" agreement $(B)$. Such an agreement generates, if implemented, an extra profit for the firms, $\pi_{B}=1$, and a negative total surplus denoted $-W_{B}$, where $W_{B}>0$ is normalized to $W_{B}=2$.

- A second category of pairs of firms can sign "good" agreements $(G)$. Such agreements, if implemented, have a positive overall effect on welfare $W_{G}=2 \omega($ with $\omega>0)$. Firms then increase their profits by $\pi_{G}=\pi<1$.

In contrast to Barros (2003) and Neven (2001), we do not want to focus on the effects of selected policies on the type of agreements chosen by the firms and thus assume that the firms cannot influence the type of agreements they may sign, but only choose whether to sign (and implement) them. We believe that this assumption is realistic in our discrete framework: although the firms might be able to make their agreement more appealing to the $C A$, this might be limited to fine-tuning.

The $C A$ does not observe the type of each agreement but knows the sizes of the two groups, i.e. the proportions of good $\left(\eta_{G}\right)$ and bad $\left(\eta_{B}\right)$ agreements. In order to simplify the presentation, we assume that these proportions are identical $\left(\eta_{G}=\eta_{B}=\frac{1}{2}\right)$. The $C A$ 's decision to prohibit an agreement will depend on these proportions, but also on the

\footnotetext{
${ }^{4}$ We consider here agreements falling under article 81(3). Agreements can either be harmful or beneficial, the latter being the most interesting type of agreements: even if they restrict competition, they may have positive effects that can offset harmful effects and increase social welfare.
} 
overall impact on welfare. Without any additional information the expected welfare is:

$$
\mathbb{E} W=\eta_{G} W_{G}-\eta_{B} W_{B}=\omega-1
$$

The variable $\omega \in[0,+\infty[$ can thus be interpreted as the $C A$ 's prior beliefs which will in practice depend on the category of agreements we consider. For instance, the $C A$ is likely to have more optimistic priors when dealing with joint research and development agreements than with horizontal market sharing or price fixing agreements.

The $C A$ 's objective is to maximize the welfare impact of the agreements, and it therefore wants to deter bad agreements while ensuring that good agreements are effectively signed. The $C A$ has to determine which policy to implement, that is, to choose when and how to control agreements. It can choose among legal regimes, which will be described in more details later on:

- Pure ex post control: Agreements are audited after they have been signed and partially implemented. This system corresponds to legal exception regime recently adopted by the European Commission.

- Mixed regime with notifications: Agreements can be monitored, either before they are actually signed by the firms (ex ante control through a notification system) or as in the pure ex post regime after they have been signed. This regime reflects the system used before the reform.

A third possibility is to consider that any agreement is legal per se and no monitoring takes place. This system corresponds exactly to the existing block exemptions regulations $(B E)$ used by the European Commission to reduce the number of notifications and save resources. Under this regime, all agreements are signed and fully implemented, and the expected social welfare is $W^{B E}=\omega-1$.

Notice that these policies cover the whole range of policies implemented by the European Commission for agreements between firms falling under article $81 .^{5}$

\section{Legal Exception Regime}

Let us first consider the case of pure ex post control or legal exception regime: Under such regime, any agreement is valid (and can thus be implemented) unless it is audited and prohibited by the $C A$. Formally, the game we analyze in this section is the following:

\footnotetext{
${ }^{5}$ As we will see later, the block exemptions and black list (i.e. all agreements are per se illegal) regimes are special cases of ex post control.
} 
1. Each pair of firms observes the type $(T \in\{B, G\})$ of the agreement they have the opportunity to sign, and decides whether to sign it $\left(s_{T}=1\right)$ or not $\left(s_{T}=0\right)$. These decisions are not observed by the $C A$.

2. The $C A$ chooses the probability $p$ with which it will run the investigations. During the audit the $C A$ receives additional information about the agreement (i.e. it receives a signal $\sigma \in\{b, g\})$ on which it can base its decision to clear $\left(\delta_{E}(\sigma)=1\right)$ or to prohibit the agreement $\left(\delta_{E}(\sigma)=0\right)$. If the $C A$ decides to prohibit the agreement, it can also impose a financial penalty that may depend on the information received during the investigation $(F(\sigma) \geq 0)$.

Let us now discuss in more details the main assumptions of this model. First, we assume that the $C A$ cannot pre-commit to the probabilities or to the decisions it will take, following the modeling strategy adopted by Besanko and Spulber (1993) in the case of mergers. This assumption has clearly implications on the equilibria. ${ }^{6}$ However, this seems consistent with the application of competition law: although the competition authorities publish guidelines on how agreements will be challenged, these guidelines are only a general set of rules leaving the authorities with some discretion when taking a decision.

Any audit takes place after the agreement has been signed and partially implemented. Therefore, when the $C A$ decides to stop an agreement after an investigation, its impact on the firms' profits and on the social welfare is only partial. We denote by $\theta$, with $0<\theta<1$, the share of the outcome (additional profits and impact on welfare) that has already been realized.

Audits are assumed to be costly and the $C A$ 's resources are supposed to be scarce so that only a maximum proportion $\beta<1$ of all potential agreements can actually be audited. When the $C A$ analyzes a case, it discovers with certainty whether an agreement has been signed or not and receives a signal $(\sigma \in\{b, g\})$, which allows it to revise its beliefs about the impact of the agreement. This signal is imperfectly correlated with the agreement's actual type and the signal's accuracy, denoted by $\rho$, with $\frac{1}{2}<\rho<1$, is assumed to be independent of the agreement's type:

$$
\operatorname{Pr}(\sigma=b \mid T=B)=\operatorname{Pr}(\sigma=g \mid T=G)=\rho .
$$

\footnotetext{
${ }^{6}$ Reinganum and Wilde $(1985 \mathrm{a}, 1986)$ in the context of tax evasion and Picard (1996) in the context of insurance fraud show that the equilibrium audit policies are strongly affected when commitment is impossible.
} 
Therefore, the risk of type I (rejecting a good agreement) and type II (accepting a bad agreement) errors, occurs with the same probability: $1-\rho$. When the signal is weakly accurate ( $\rho$ close to $\frac{1}{2}$ ), the $C A$ will then take a decision based mostly on its prior beliefs, while if the signal is very accurate ( $\rho$ close to 1 ) this decision will be based on an almost perfect knowledge and errors are then unlikely to occur.

Finally, we assume that the fines that can be imposed by the $C A$ are capped and denote by $\bar{F}$ the maximum possible fine. In the European Union for instance, fines are limited to $10 \%$ of the annual turnover of the involved firms.

Let us now show that a black list regime can be effective if $\bar{F}$ is high enough. Suppose that the $C A$ sets a probability of audit equal to $\beta$, decides to prohibit any agreement, irrespective of the signal received, and sets a fine equal to $\bar{F}$. The expected profit generated by a bad agreement is then:

$$
\mathbb{E} \Pi_{B}=1-\beta(1-\theta)-\beta \bar{F}
$$

Therefore, if $\bar{F}$ is high enough, bad agreements (and thus good agreements as well) are deterred. Note that because we have modeled the audit cost as a sunk cost, this remains an equilibrium even when the $C A$ cannot commit to a specific policy. In the rest of the paper we will assume that:

$$
\bar{F}>\frac{1-\beta(1-\theta)}{\beta},
$$

so that the black list regime is effective in the sense that there always exists an equilibrium for which no agreement is signed and the expected welfare is $W^{B L}=0 .^{7}$

Let us now analyze the perfect Bayesian equilibria of the ex post control game. As usual in this type of situation, multiple equilibria might co-exist. We focus on the $C A$ 's preferred equilibrium, i.e., the equilibrium generating the highest expected welfare and only look for equilibria that yield an outcome differing from both block exemptions and black list. ${ }^{8}$ Ajouter Référence (pour le choix de l'équilibre).

Consider first the optimal decisions that should be taken by the $C A$ given the firms' equilibrium decisions $\left(s_{B}^{*}, s_{G}^{*}\right) \neq(0,0)$. If, when auditing, the signal received is bad

\footnotetext{
${ }^{7}$ Note that if the cost of auditing was not sunk, for any $\varepsilon>0$, we could have an equilibrium for which the expected welfare is $-\varepsilon$. This would be the case for

$$
s_{G}^{*}=0, s_{B}^{*}=\frac{\varepsilon}{1-\beta(1-\theta)}, p^{*}=\beta \text { and } F^{*}(b)=F^{*}(g)=\frac{1-\beta(1-\theta)}{\beta} .
$$

${ }^{8}$ Note that block exemptions only make sense for agreements with positive expect welfare $(\omega \geq 1)$, while black list is relevant for agreements with negative expected welfare $(\omega \leq 1)$.
} 
$(\sigma=b)$, the $C A$ updates its beliefs:

$$
\operatorname{Pr}(B \mid b) \equiv \operatorname{Pr}(T=B \mid \sigma=b)=\frac{\rho s_{B}^{*}}{\rho s_{B}^{*}+(1-\rho) s_{G}^{*}},
$$

and therefore prohibits the agreements $\left(\delta_{E}^{*}(b)=0\right.$, possibly combined with a fine $\left.F(b)\right)$ whenever the expected welfare is negative, i.e. when:

$$
\begin{aligned}
\operatorname{Pr}(G \mid b) W_{G}-\operatorname{Pr}(B \mid b) W_{B} \leq 0 & \Leftrightarrow \operatorname{Pr}(B \mid b) \geq \operatorname{Pr}(G \mid b) \omega \\
& \Leftrightarrow s_{B}^{*} \geq \frac{(1-\rho) \omega}{\rho} s_{G}^{*} .
\end{aligned}
$$

Similarly, the $C A$ prohibits an agreement for which it has received a good signal $(\sigma=g)$ whenever:

$$
\operatorname{Pr}(G \mid g) W_{G}-\operatorname{Pr}(B \mid g) W_{B} \leq 0 \Leftrightarrow s_{B}^{*} \geq \frac{\rho \omega}{1-\rho} s_{G}^{*} .
$$

Given that $\rho>\frac{1}{2}$, it is impossible to have $\delta_{E}^{*}(g)=0$ and $\delta_{E}^{*}(b)=1$ and therefore only three combinations of decisions are possible. The following lemma shows that only one case is relevant for our analysis.

Lemma 1 For the outcome of a perfect Bayesian equilibrium of the ex post control game to be preferred to the block exemptions and black list regimes, it must be the case that the $C A$ prohibits an agreement if and only if it has received a bad signal, that is, $\delta_{E}^{*}(g)=1$ and $\delta_{E}^{*}(b)=0$.

Proof. Consider first an equilibrium for which the $C A$ clears any agreement whatever the signal received during the audit. This would be anticipated by the firms and all agreements would then be signed. Therefore such an equilibrium is outcome-equivalent to the block exemptions.

Consider now an equilibrium for which the $C A$ investigates with probability $p$ and prohibits any agreement whatever the signal received. In this case the expected welfare is:

$$
W=(1-p(1-\theta))\left(\omega s_{G}-s_{B}\right) .
$$

But for such an equilibrium to exist it must be the case that: $s_{B} \geq \frac{\rho \omega}{1-\rho} s_{G}>\omega s_{G}$, and therefore $W<0$. Such an equilibrium would thus be strictly dominated by the black list equilibrium.

We now focus on equilibria for which an agreement is prohibited if (and only if) it is investigated and the signal is bad. This is optimal when:

$$
\frac{(1-\rho) \omega}{\rho} \leq \frac{s_{B}^{*}}{s_{G}^{*}} \leq \frac{\rho \omega}{1-\rho} .
$$


Note now that in this ex post control setting, the threat of having to pay an hefty fine influences the firms' decisions to sign an agreement. Optimally, the $C A$ would like to deter the bad agreements while ensuring that all good agreements are signed. However, since it cannot credibly commit to a particular audit and decisions' policy, such separating equilibria cannot exist. At best, the $C A$ might be able to ensure that all beneficial agreements are signed while deterring some of the bad ones. Besides, this will only be possible if the signal received during the audit is accurate enough, since the extra profit generated by a bad agreement is larger than the profit created by a good agreement. The firms' expected profits when they sign are:

$$
\mathbb{E} \Pi_{B}=1-p \rho(1-\theta)-p \rho F(b) \text { and } \mathbb{E}_{G}=(1-p(1-\rho)(1-\theta)) \pi-p(1-\rho) F(b)
$$

For bad agreements to be partially deterred while good agreements are signed, it must be the case that $\mathbb{E} \Pi_{B}=0$ and $\mathbb{E} \Pi_{G} \geq 0$, that is:

$$
F(b)=\frac{1-p \rho(1-\theta)}{p \rho} \leq \frac{(1-p(1-\rho)(1-\theta)) \pi}{p(1-\rho)} .
$$

This requires to have:

$$
1-\rho(1+\pi) \leq p(1-\theta) \rho(1-\rho)(1-\pi)
$$

Condition (1) is fulfilled for sufficiently high values of $\rho \geq \widehat{\rho}(p, \theta, \pi)$, where $\widehat{\rho}(p, \theta, \pi)$ is the unique value of $\rho$ within the interval $] \frac{1}{2} ; 1$ [ such that the left-hand and right-hand terms in equation (1) are equal. Note that the condition (1) and the required level of fine are independent of $s_{B}^{*}$. Given that the expected welfare is a strictly decreasing function of $s_{B}^{*}$, the "semi-separating" equilibrium that will be preferred by the $C A$ is such that $s_{B}^{*}=\frac{(1-\rho) \omega}{\rho}$ and this is feasible (i.e. $s_{B}^{*}$ is lower than 1 ) whenever $\omega<\frac{\rho}{1-\rho}$. In this case, the expected welfare writes:

$$
W_{S S}^{E}=(1-p(1-\rho)(1-\theta)) \omega-(1-p \rho(1-\theta)) \frac{(1-\rho) \omega}{\rho}=\frac{2 \rho-1}{\rho} \omega .
$$

Note that this is independent of $p$. However, because both the fine and $\widehat{\rho}(p, \theta, \pi)$ are decreasing functions of $p$ this equilibrium is most likely to exist when $p^{*}=\beta$. This is possible only if $\bar{F}$ is large enough:

$$
\bar{F} \geq \frac{1-\beta \rho(1-\theta)}{\beta \rho} \Leftrightarrow \rho \geq \bar{\rho}(\bar{F}, \beta, \theta) \equiv \frac{1}{\beta(\bar{F}+1-\theta)} .
$$

If one of the two conditions (1) or (2) is not satisfied, that is, if

$$
\rho<\rho^{E}(\beta, \theta, \bar{F}, \pi) \equiv \max [\widehat{\rho}(\beta, \theta, \pi), \bar{\rho}(\beta, \theta, \bar{F})]
$$


this semi-separating equilibrium does not exist. The only other possible equilibrium that generates a different outcome from both block exemptions and black list is then a "pooling" equilibrium for which all agreements are signed. In this case, the probability of audit is equal to $\beta$ and the penalty has to be low enough (e.g. equal to 0 ). This equilibrium exists if and only if:

$$
\frac{(1-\rho) \omega}{\rho} \leq 1 \leq \frac{\rho \omega}{1-\rho} \Leftrightarrow \frac{(1-\rho)}{\rho} \leq \omega \leq \frac{\rho}{1-\rho} .
$$

In this case the expected welfare is:

$$
\begin{aligned}
W_{P}^{E} & =(1-\beta(1-\rho)(1-\theta)) \omega-(1-\beta \rho(1-\theta)) \\
& =\omega-1+\beta(1-\theta)(\rho-(1-\rho) \omega) .
\end{aligned}
$$

Finally, we have:

$$
W_{P}^{E}>0 \Leftrightarrow \omega>\omega_{P}^{E}(\rho, \beta, \theta) \equiv \frac{1-\beta \rho(1-\theta)}{1-\beta(1-\rho)(1-\theta)} .
$$

The results of this analysis are summarized in the following proposition and illustrated in Figure 1:

Proposition 1 The perfect Bayesian equilibrium of the pure ex post game that is preferred by the CA, dominates the block exemptions regime if and only if $\omega<\frac{\rho}{1-\rho}$. In this case, we have:

- If $\rho \geq \rho^{E}(\beta, \theta, \bar{F}, \pi)$, the preferred equilibrium is the best semi-separating equilibrium $\left(E_{S S}\right)$ for which the expected welfare is equal to $W_{S S}^{E}=\frac{2 \rho-1}{\rho} \omega$.

- If $\rho<\rho^{E}(\beta, \theta, \bar{F}, \pi)$ and $\omega>\omega_{P}^{E}(\rho, \beta, \theta)$, the preferred equilibrium is the "pooling" equilibrium $\left(E_{P}\right)$ in which all agreements are signed. The expected welfare is then:

$$
W_{P}^{E}=\omega-1+\beta(1-\theta)(\rho-(1-\rho) \omega) .
$$

- If $\rho<\rho^{E}(\beta, \theta, \bar{F}, \pi)$ and $\omega \leq \omega_{P}^{E}(\rho, \beta, \theta)$, then the black list regime is preferred and the expected welfare is $W^{B L}=0$.

When the signal is sufficiently accurate, it is possible to deter some of the bad agreements while ensuring that all good ones are signed. Moreover, for the best of these equilibria the proportion of bad agreements which are signed is low enough $\left(s_{B}^{*}=\frac{(1-\rho) \omega}{\rho}<1\right)$ so that the expected welfare is strictly positive. Thus, the semi-separating equilibrium 


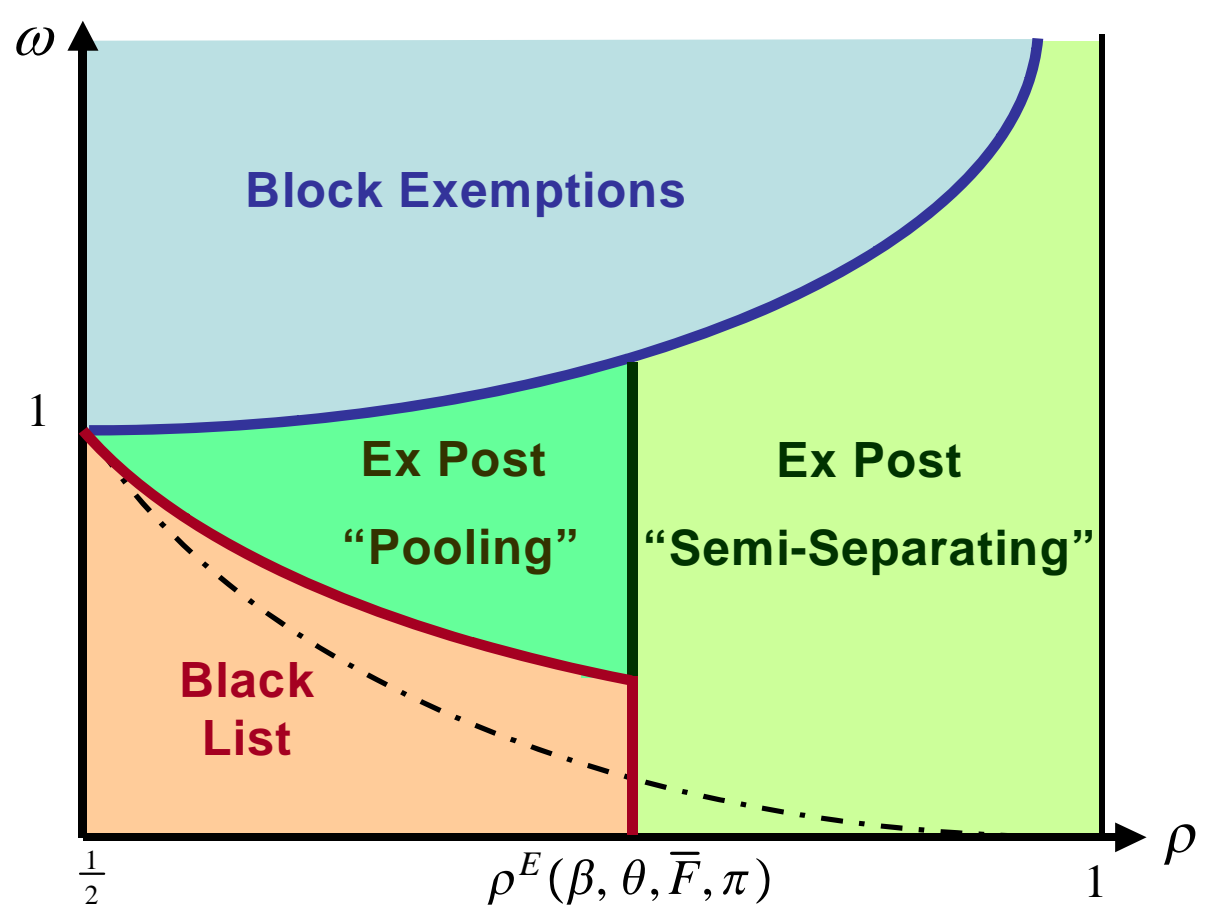

Figure 1: Optimal Ex Post Control

always dominates the black list regime. However, type I errors (i.e. prohibiting good agreements) occur and the cost of these errors increases with $\omega$. When priors are very optimistic $\left(\omega \geq \frac{\rho}{1-\rho}\right)$, type I errors are too costly relatively to type II errors (i.e. allowing bad agreements) and the block exemptions regime is preferred. Notice however, that when the signal becomes more accurate, errors become less likely and the semi-separating equilibrium is more often preferred.

When the signal is not accurate enough, it is no longer possible to obtain a semiseparating equilibrium. The choice is now between the two per se regimes and a pooling equilibrium in which all agreements are signed but some type I and II errors will be made. We thus have an arbitrage between type I and type II errors: in the black list (resp. block exemptions) regime the $C A$ will only make type I (resp. type II) errors, while in the pooling equilibrium type I as well as type II errors occur with the same probability: $1-\rho$. When $\omega$ is low type I errors are not costly but type II errors are. The opposite is true when $\omega$ is high. Therefore, black list is preferred for pessimistic priors in order to limit type II errors, block exemptions is chosen when priors are optimistic to eliminate costly type I errors, while the pooling equilibrium will be optimal for intermediate values of $\omega$.

Note also that $\rho^{E}(1, \theta, \bar{F}, \pi)>\frac{1}{2}$. Even if the $C A$ was able to monitor all potential agreements, a semi-separating equilibrium would not always exist: the problem comes 
from the risk of error not from the fact that some agreements are not monitored.

\section{Mixed Regime with Notifications}

One of the problems when controlling agreements ex post is that part of the impact on the welfare has already been realized. It would be preferable to be able to stop bad agreements earlier in order to avoid the negative impact on welfare. One possibility is to require that firms notify their agreement in order to obtain negative clearance before implementing it. However, this ex ante monitoring can only work if the $C A$ is also able to intervene ex post in order to control (and if necessary prohibit and punish) agreements that would have been signed but not notified. We now look at such a mixed regime of monitoring that uses both notifications and ex post control and analyze the following extension of the ex post control game:

1. Pairs of firms observe the type $(T \in\{B, G\})$ of agreements that they have the opportunity to sign. They then decide whether to notify the agreement $\left(n_{T}=1\right)$, to sign it without notifying $\left(s_{T}=1\right)$ or to reject it $\left(r_{T}=1\right)$. The $C A$ only observes the mass ("number") of agreements that have been notified.

2. The $C A$ decides how to allocate its budget between ex ante and ex post control: it chooses which proportion of the potential agreements to control ex ante $(a)$ or ex post (e, with $a+e \leq \beta)$.

(a) $\boldsymbol{E x}$ ante control: When an agreement is investigated, the $C A$ receives an imperfect signal $\left(\sigma_{N} \in\{b, g\}\right)$. It must then decide whether to allow the agreement or not $\left(\delta_{N}\left(\sigma_{N}\right) \in\{0,1\}\right)$. It also has to decide whether to allow agreements that have not been audited $\left(\delta_{N}(\emptyset) \in\{0,1\}\right)$. Agreements that are allowed are then implemented while agreements that have been blocked are abandoned.

(b) Ex post control: This stage is similar to the second stage of the ex post control game, that is, the $C A$ takes a decision based on the signal received during the audit $\left(\delta_{E}\left(\sigma_{E}\right) \in\{0,1\}\right)$ and, when relevant, chooses the fine $\left(F\left(\sigma_{E}\right) \geq 0\right)$.

We assume that notifications differ from ex post control in three aspects:

- As already mentioned, monitoring takes place before the firms actually sign their agreements and therefore if the $C A$ decides to block an agreement the impact on the firms' profit and welfare is null. 
- In order to be consistent with the European Commission's practice, we assume that no fine can be imposed if a notified agreement is blocked. We believe it to be a reasonable assumption since the agreements have not yet been signed or implemented. Imposing fines could therefore be seen as taxing firms for the right to sign and implement an agreement, which is far beyond the aim of competition policy.

- Finally, whereas in the ex post control, agreements that are not investigated are cleared, we assume that at the notification stage, the $C A$ can always block an agreement without investigating. In practice, the Commission cannot forbid an agreement without analyzing it (firms would otherwise be able to complain in front of a Court), however it can always delay its decisions, so that the agreement is de facto prohibited. ${ }^{9}$

The notification stage is identical to the ex post control in all other aspects. In particular, we assume that the audit technology is the same (that is, $\rho_{N}=\rho_{E}=\rho$ ) and the maximum proportion of potential agreements that can be audited is again equal to $\beta .^{10}$ We appreciate that these conditions are unlikely to be satisfied in practice: for instance, a notification is costly for the firm that has to provide the European Commission with detailed information about the agreement and the relevant market, whereas an ex post investigation requires a longer and more difficult information acquisition process for the Commission. However, our objective is to analyze the trade-off between a preventive policy (notification)and a repression regime with fines (legal exception). We thus choose to limit the differences between the two regimes to what we see as the essential features (timing and fines) in order to be able to distinguish the effects of each instrument and keep the model as simple as possible.

\subsection{Preliminary Results}

Characterizing and comparing the outcome of all the equilibria of this game is cumbersome and we thus limit ourselves to identifying the values of the parameters for which the use of notifications is optimal (i.e. increases the expected welfare compared to the ex post control framework).

\footnotetext{
${ }^{9}$ According to Neven, Papandropoulos and Seabright (1998), the average length of the notification process depends on the type of decision but is always longer than a year: 13 months for a settlement, 20 months for a comfort letter, 34 months to be granted a formal exemption and 44 months for a formal prohibition.

${ }^{10}$ This is equivalent to assuming that the costs of an audit ex ante and ex post are the same.
} 
Lemma 2 There is no perfect Bayesian equilibrium that strictly dominates the preferred equilibrium of the pure ex post control game for which either no agreement is notified $\left(n_{B}^{*}=n_{G}^{*}=0\right)$ or no agreement is signed $\left(s_{B}^{*}=s_{G}^{*}=0\right)$.

Proof. Any equilibrium such that $n_{B}^{*}=n_{G}^{*}=0$ is equivalent to one equilibrium of the pure ex post control game. This implies the first part of the proof. Suppose now that there exists an equilibrium for which $s_{B}^{*}=s_{G}^{*}=0$. Because the expected profit of a firm that notifies cannot be negative, it must be the case that all firms notify or that the expected welfare of notifying is exactly 0 . However, if this is the case, the outcome is equivalent to black list. Finally, if all agreements are notified, either $\omega$ is such that agreements are never blocked in which case the equilibrium is equivalent to the block exemptions, or the $C A$ allocates all of its budget to the treatment of the notifications and the probability of ex post control is 0 . However, one firm would then find it profitable to deviate and sign without notifying, since this deviation would not be detected.

As a consequence, we only consider equilibria for which some agreements but not all are notified, that is, we have $0<n^{*}<1$, where $n^{*}=\frac{n_{B}^{*}+n_{G}^{*}}{2}$ is the mass of notified agreements. The $C A$ 's optimal decision when monitoring takes place once agreements have been signed are identical to what they were in the pure ex post control case, that is, audited agreements are prohibited whenever:

- $s_{B}^{*} \geq \frac{(1-\rho) \omega}{\rho} s_{G}^{*}$, if the $C A$ has received a bad signal $\left(\sigma_{E}=b\right)$;

- $s_{B}^{*} \geq \frac{\rho \omega}{1-\rho} s_{G}^{*}$, if the $C A$ has received a good signal $\left(\sigma_{E}=g\right)$.

The analysis of the optimal decisions for ex ante control is very similar. On the basis of the signal it has received during the investigation, the $C A$ updates its beliefs exactly as in the ex post case and therefore it decides to block an agreement for which the signal is:

- $\operatorname{bad}\left(\delta_{N}(b)=0\right)$ whenever $n_{B}^{*} \geq \frac{(1-\rho) \omega}{\rho} n_{G}^{*}$;

- $\operatorname{good}\left(\delta_{N}(g)=0\right)$ whenever $n_{B}^{*} \geq \frac{\rho \omega}{1-\rho} n_{G}^{*}$.

When it does not investigate, it bases its decisions on its priors and therefore blocks the agreement $\left(\delta_{N}(\emptyset)=0\right)$ whenever $n_{B}^{*} \geq \omega n_{G}^{*}$.

Combining all of this means that we potentially have 12 cases for the optimal decision profile $\Delta^{*}$, where:

$$
\Delta^{*}=\left(\Delta_{N}^{*}, \Delta_{E}^{*}\right), \text { with } \Delta_{N}^{*}=\left(\delta_{N}^{*}(g), \delta_{N}^{*}(b), \delta_{N}^{*}(\emptyset)\right) \text { and } \Delta_{E}^{*}=\left(\delta_{E}^{*}(g), \delta_{E}^{*}(b)\right)
$$


Some of them can however be ruled out: for instance, the outcome of any equilibrium for which either $\Delta_{N}^{*}=(1,1,1)$ or $\Delta_{E}^{*}=(1,1)$ is equivalent to the block exemptions regime. We also rule out any equilibrium for which $\Delta_{N}^{*}=(0,0,0)$. In this case, firms are actually indifferent between notifying their agreement or refusing to do it and we then assume that firms prefer not to notify. ${ }^{11}$ Thus, any equilibrium for which $\Delta_{N}^{*}=(0,0,0)$ corresponds to an equilibrium of the pure ex post game. This means that we are left with equilibria for which the expected profit of a firm that notifies is strictly positive. In equilibrium we must thus have $n_{T}^{*}+s_{T}^{*}=1$ for any $T \in\{B, G\}$.

We can therefore restrict our attention to the following four types of equilibria: ${ }^{12}$

$N_{1}: \Delta^{*}=((1,0,0),(0,0))$ for $\omega n_{G}^{*} \leq n_{B}^{*} \leq \frac{\rho \omega}{1-\rho} n_{G}^{*}$ and $s_{B}^{*} \geq \frac{\rho \omega}{1-\rho} s_{G}^{*}$.

$N_{2}: \Delta^{*}=((1,0,1),(1,0))$ for $\frac{(1-\rho) \omega}{\rho} n_{G}^{*} \leq n_{B}^{*} \leq \omega n_{G}^{*}$ and $\frac{(1-\rho) \omega}{\rho} s_{G}^{*} \leq s_{B}^{*} \leq \frac{\rho \omega}{1-\rho} s_{G}^{*}$.

$N_{3}: \Delta^{*}=((1,0,0),(1,0))$ for $\omega n_{G}^{*} \leq n_{B}^{*} \leq \frac{\rho \omega}{1-\rho} n_{G}^{*}$ and $\frac{(1-\rho) \omega}{\rho} s_{G}^{*} \leq s_{B}^{*} \leq \frac{\rho \omega}{1-\rho} s_{G}^{*}$.

$N_{4}: \Delta^{*}=((1,0,1),(0,0))$ for $\frac{(1-\rho) \omega}{\rho} n_{G}^{*} \leq n_{B}^{*} \leq \omega n_{G}^{*}$ and $s_{B}^{*} \geq \frac{\rho \omega}{1-\rho} s_{G}^{*}$.

Note that none of these equilibria exist when the priors are too optimistic $\left(\omega \geq \frac{\rho}{1-\rho}\right)$. In this case, block exemption remains the optimal outcome. The main reason is that notifications cannot be used to reduce drastically the number of type I errors. (A revoir???)

Let us now assume that $\omega<\frac{\rho}{1-\rho}$ and compare the type $N_{i}$ equilibria to the semiseparating equilibrium $\left(E_{S S}\right)$ of the ex post control game. A first major difference is that it is no longer possible to (partially) deter bad agreements: they will now be either notified or signed without notifying. Moreover, because the expected profit of a firm that notifies is strictly positive, it must be the case that in equilibrium ex post control is now less efficient (in the sense that the deterrence effect is lower) than in the pure ex post control case. Therefore, type II errors are likely to occur more often than in the semi-separating equilibrium $E_{S S}$ and type I errors will be more frequent.

\footnotetext{
${ }^{11}$ Without this assumption we could generate equilibria that dominate any equilibrium of the pure ex post game. However, this would be very artificial since it would only come from the fact that some firms notify their agreement although they anticipate that their agreement will be blocked without being audited. Moreover, these equilibria would not be robust to the introduction of an (even) infinitesimal cost of notification.

${ }^{12} \mathrm{~A}$ preliminary analysis of these different types of equilibria is provided in appendix $A$. It will be used in the proofs of the results presented in the rest of the paper.
} 
Consider for instance the type $N_{2}$ equilibrium for which the decisions are ex ante and ex post identical to the decisions taken in the $E_{S S}$ equilibrium: because all the firms must be indifferent between signing and notifying, in equilibrium, the fine must be $F(b)=0$ and the audit probabilities must be such that $p_{N}=(1-\theta) p_{E}$. This also implies that the probability of audit ex post will now be larger than $\beta$ and therefore both types of errors will be more frequent.

A similar analysis holds for the other types and leads to the following proposition:

Proposition 2 Whenever the "semi-separating" equilibrium $\left(E_{S S}\right)$, in which all the good agreements and part of the bad agreements are signed, exists, it is preferred by the CA to any equilibrium involving notifications.

\section{Proof. See Appendix B.}

Consider now the case of pessimistic priors $\left(\omega \leq \frac{1-\rho}{\rho}\right)$ and compare the types $N_{1}$ and $N_{4}$ equilibria to the black list regime. In both types $N_{1}$ and $N_{4}$ equilibria the number of type I errors will be reduced but this positive impact will remain limited since $\omega$ is low. On the other hand, type II errors - which are now relatively more costly than type I errors - occur since the $C A$ (at least) clears the agreements for which it has received a good signal when treating the notification. Moreover, because the expected profits of a firm that notifies is strictly positive, the probability of ex post audit cannot be too large thereby increasing substantially the number of type II errors. This is made even worse when the $C A$ decides to clear notified agreements that have not been audited (e.g. $N_{4}$ ). This analysis leads to the following proposition:

Proposition 3 When the priors are pessimistic, that is when $\omega \leq \frac{1-\rho}{\rho}$, the black list equilibrium is preferred by the CA to any equilibrium involving notifications.

\section{Proof. See Appendix C.}

\subsection{When Is the Use of Notifications Optimal?}

In this subsection, following the results of proposition 2 and 3, we restrict our attention to intermediate priors, $\left(\frac{1-\rho}{\rho}<\omega<\frac{\rho}{1-\rho}\right)$ and look for equilibria involving notifications that are preferred by the $C A$ to the pooling equilibrium $\left(E_{P}\right)$ of the pure ex post control game. 
In order to better understand the type of equilibria that are likely to meet this criterion, suppose first that the $C A$ can ensure that all agreements are notified. The $C A$ 's equilibrium strategy is then extremely simple: notifications are investigated with probability $p_{N}=\beta$ and the $C A$ follows the signal received during the investigation, i.e., agreements are blocked if and only if the signal is bad. Finally, it follows its priors for agreements that are not investigated. The expected welfare is then equal to:

$$
\begin{cases}W_{L}=\beta(\rho \omega-1+\rho) & \text { for } \frac{1-\rho}{\rho}<\omega \leq 1, \\ W_{H}=\omega-1+\beta(\rho-(1-\rho) \omega) & \text { for } 1<\omega<\frac{\rho}{1-\rho} .\end{cases}
$$

Now remember that in the pooling equilibrium $\left(E_{P}\right)$, the expected welfare is

$$
W_{P}^{E}=\omega-1+\beta(1-\theta)(\rho-(1-\rho) \omega)
$$

Notice first that we have $W_{H}>W_{P}^{E}$. This result is intuitive: The policies implemented in these two equilibria are identical, but intervention occurs earlier in the notification regime, i.e., before the agreements are partially implemented. The situation is even more favorable to notifications when the priors are pessimistic $(\omega<1)$ : not only it is better to intervene early but, given that the priors are rather pessimistic, it is better to block agreements that are not investigated. Because agreements can now be blocked without auditing, these equilibria also dominate the black list regime.

Unfortunately, in the framework with endogenous notifications and without commitment an equilibrium in which all agreements are notified never exists. However, as lemmas 3 and 4 show, it is sometimes possible to find equilibria that are very similar to the "exogenous notification equilibrium":

Lemma 3 There exists a threshold $\bar{\omega}_{1}(\rho, \theta) \equiv \min \left[1, \frac{1-\rho+2(1-\theta)}{\rho}\right]$ such that for $\theta \geq \beta(1-\rho)$, $\omega<\bar{\omega}_{1}(\rho, \theta)$ and $\varepsilon>0$ small enough, there exists a type $N_{1}$ equilibrium with $n_{G}^{*}=1$ and $n_{B}^{*}=1-2 \varepsilon$ that is preferred by the $C A$ to the pooling and the black list equilibria of the ex post control game.

\section{Proof. See Appendix D.}

If the $C A$ was able to commit to its audit and decision strategies, setting $e=\beta-a=\varepsilon$ and high enough fines (e.g. equal to $\bar{F}$ ) would ensure that all agreements would be notified and we could thus have an outcome identical to the case of "exogenous notifications." This is no longer the case without commitment but, as lemma 3 shows, it is still possible to ensure that almost all firms notify. Set for instance the "unconditional" fines 
$(F(b)=F(g)=F)$ such that the bad firms are indifferent between signing and notifying, that is:

$$
p_{N}(1-\rho)=1-(1-\theta) p_{E}-p_{E} F \Leftrightarrow F=\theta-\frac{\beta-\varepsilon}{1-\varepsilon}(1-\rho) \text {. }
$$

This is feasible whenever $\theta \geq \beta(1-\rho)$, i.e., as long as the penalty is non negative and will also guarantee that good agreements are all notified. Given that priors are rather pessimistic $(\omega \leq 1)$, it is optimal for the $C A$ to prohibit an agreement unless it has been notified, audited and led to a good signal.

To be an equilibrium, we must show that the budget allocation is optimal: allocating more resources to the ex post control would only reduce the expected welfare since $p_{E}$ would not be affected but $p_{N}$ would decrease. Consider now a marginal reallocation towards ex ante control $(d a=-d e>0)$. This has several effects: firstly, it increases the effectiveness of ex ante control, and the corresponding benefit is almost $(\rho \omega-1+\rho) d a$. However, it also leads to a greater number of type II errors ex post: although it applies to a small number of cases $(\varepsilon)$, the change in the probability of audit is important $\left(\frac{d a}{\varepsilon}\right)$. Nevertheless, because monitoring takes place rather late, the marginal cost of increasing $a$ is lower when $\theta$ is large and is only equal to $-2(1-\theta) d a$. Therefore, the proposed budget allocation is indeed optimal whenever $\omega$ is small enough, hence defining the threshold $\bar{\omega}_{1}(\rho, \theta)$.

Lemma 4 There exists a threshold $\underline{\omega}_{2}(\rho, \theta) \in\left[1, \frac{\rho}{1-\rho}\left[\right.\right.$ such that for any $\omega>\underline{\omega}_{2}(\rho, \theta)$, $\beta<1-\theta$ and any $\varepsilon>0$ small enough, there exists a type $N_{2}$ equilibrium with $n^{*}=1-\varepsilon$ that is preferred by the CA to the pooling and the black list equilibria of the pure ex post game.

\section{Proof. $\quad$ See Appendix E.}

Lemma 4 is the counterpart of lemma 3 for optimistic priors (i.e., $\omega \geq 1$ ), and shows that it is sometimes possible to achieve an outcome (almost) equivalent to the outcome of the exogenous notification equilibrium. Consider now a type $N_{2}$ equilibrium for which the audit probabilities are $p_{N}^{*}=(1-\theta) p_{E}^{*} \simeq \beta$ and the fine is $F^{*}(b)=0$. In this case, all firms are indifferent between signing and notifying the agreement. Note that this is possible only when $\beta$ is small enough, i.e., $\beta<1-\theta$.

Once again, to be an equilibrium, the impact of any marginal reallocation of the auditing budget must be equal to 0 . Recalling that a type $N_{2}$ equilibrium is such that an 
agreement is valid unless it has been audited and the audit revealed a bad signal, let us rewrite the expected welfare as a function of $a$ :

$$
W_{2}^{N}(a)=\omega-1+\left(\rho n_{B}-(1-\rho) \omega n_{G}\right) \frac{a}{n}-(1-\theta)\left(\rho s_{B}-(1-\rho) \omega s_{G}\right) \frac{\beta-a}{1-n},
$$

and therefore the equilibrium must necessarily be such that:

$$
\left(W_{2}^{N}\right)^{\prime}=0 \Leftrightarrow \frac{\rho n_{B}-(1-\rho) \omega n_{G}}{n}=\frac{(1-\theta)\left(\rho s_{B}-(1-\rho) \omega s_{G}\right)}{1-n} .
$$

This condition states that the marginal benefit of making ex ante control more effective must be equal to the marginal cost of making ex post control less effective and is true for any type $N_{2}$ equilibrium. This equation sets a condition that has to be satisfied by $n_{B}$ and $n_{G}$ and is in general quite complex. Let us however look at extreme values of $\theta$.

Consider first type $N_{2}$ equilibria for which $n^{*}=1-\varepsilon$. Condition (3) then rewrites as:

$$
\left(\rho s_{B}-(1-\rho) \omega s_{G}\right)(1-\theta n)=(\rho-(1-\rho) \omega)(1-n) .
$$

This condition is violated when $\theta$ is close to 1 . This is not surprising since the benefits of auditing ex post are close to 0 , and it is thus preferable to increase the probability of audit $e x$ ante as much as possible. Suppose now that $\theta=0$. In that case, equation (4) boils down to $s_{B}=s_{G}$ : the $C A$ 's policy to prohibit the agreements only when the signal received is bad is consistent for any priors. There thus exists a threshold $\bar{\theta}_{2}$, such that such an equilibrium leading to an outcome almost equal to $W_{H}$, exists for any $\theta \leq \bar{\theta}_{2}$. Note finally that when $\omega=\frac{\rho}{1-\rho}$, condition (4) is again $s_{B}=s_{G}$, whereas when $\omega=1$, it writes as:

$$
\left(2 \rho(1-n)-s_{G}\right)(1-\theta n)=(2 \rho-1)(1-n) \Leftrightarrow \frac{s_{B}}{s_{G}} \simeq \frac{1+\theta(2 \rho-1)}{1-2 \rho \theta} .
$$

When $\theta$ is large, i.e. when the proportion of the impact of the implemented agreements on welfare is important, this last condition requires to have $\frac{s_{B}}{s_{G}}$ very large (or even negative which is clearly impossible) and this would not be consistent with the $C A$ 's decision rule which requires to have $\frac{1-\rho}{\rho} \leq \frac{s_{B}}{s_{G}} \leq \frac{\rho}{1-\rho}$. Therefore, $\bar{\theta}_{2}$ is increasing in $\omega$ or alternatively condition (3) is met when $\omega$ is large but is unlikely to be satisfied (unless $\theta$ is close to 0 ) when $\omega$ is close to 1 ; this defines the threshold $\underline{\omega}_{2}(\rho, \theta)$.

Consider now a type $N_{2}$ equilibrium in which almost all agreements are signed without being notified, i.e. $n^{*}=\varepsilon$. Most of the analysis we just have done for $n^{*}=1-\varepsilon$ remains valid and condition (3) now writes as:

$$
\frac{\rho n_{B}-(1-\rho) \omega n_{G}}{\rho-(1-\rho) \omega}=\frac{(1-\theta) n}{1-n} .
$$


This condition is now satisfied even for $\theta=1$. Such an equilibrium is thus likely to exist for any $\omega$. Moreover, because some agreements are notified, this equilibrium is preferred to the pooling equilibrium $\left(E_{P}\right)$ : decisions are indeed the same, audit probabilities are almost identical but some of the agreements are monitored earlier which is always preferable. However, because it is very similar to the "pooling" equilibrium $\left(E_{P}\right)$ it is dominated by the black list equilibrium if $\omega$ is too small. This analysis leads to the following result:

Lemma 5 There exists a threshold $\left.\widetilde{\omega}_{2}(\rho, \beta, \theta) \in\right] \frac{1-\rho}{\rho}, 1\left[\right.$ such that for any $\omega>\widetilde{\omega}_{2}(\rho, \beta, \theta)$ and any $\varepsilon>0$ small enough, there exists a type $N_{2}$ equilibrium with $n^{*}=\varepsilon$ that is preferred by the CA to the pooling equilibrium of the pure ex post game.

\section{Proof. $\quad$ See Appendix E.}

We have focused here on extreme values, searching only for equilibria for which either almost all agreements are notified, or almost all agreements are signed but not notified. For some values of the parameters of the model, numerous other equilibria exist: for instance, for values of $\omega$ larger than 1 (but also for lower values when $\theta$ is not too large), it is possible to show that there exists equilibria for any $n^{*} \in[0, \beta]$. Often these equilibria will also be preferred to the "pooling" $\left(E_{P}\right)$ and the black list equilibria.

We can now combine these different results to understand when notifications are optimal. Lemmas 3 and 5 lead to the following proposition:

Proposition 4 Assume that $\theta>\frac{\beta}{2}$. The $C A$ 's preferred equilibrium involves notifications whenever $\rho<\rho^{E}(\beta, \theta, \bar{F}, \pi)$ and $\omega_{P}^{E}(\rho ; \beta, \theta)<\omega<\frac{\rho}{1-\rho}$. Moreover, there exists a threshold $\left.\bar{\theta}^{N}(\beta, \bar{F}, \pi) \in\right] \frac{1}{2}, 1\left[\right.$, such that for any $\theta<\bar{\theta}^{N}(\beta, \bar{F}, \pi)$, the CA's preferred equilibrium involves notifications if and only if $\rho<\rho^{E}(\beta, \theta, \bar{F}, \pi)$ and $\left.\omega \in\right] \frac{1-\rho}{\rho}, \frac{\rho}{1-\rho}[$.

\section{Proof. See Appendix F.}

Lemma 3 shows that an equilibrium for which almost all agreements are notified exists whenever the impact of the implemented agreements is important, i.e., $\theta \geq \beta(1-\rho)$ and when the priors are pessimistic, i.e., $\omega<\bar{\omega}_{1}(\rho, \theta)$. For values of $\theta$ low enough, $\bar{\omega}_{1}(\rho, \theta)=1$ for any accuracy of the signal low enough, i.e., $\rho<\rho^{E}(\beta, \theta, \bar{F}, \pi)$. Therefore, notifications will always be optimal for intermediate priors: $\left.\omega \in] \frac{1-\rho}{\rho}, 1\right]$ when $\theta$ and $\rho$ are low enough. Moreover, lemma 5 shows that an equilibrium with notification exists and is preferred by 
the $C A$ to any equilibrium of the pure ex post game whenever $\omega>\widetilde{\omega}_{2}(\rho, \beta, \theta)$. Given that this threshold is always lower than 1 , notifications are always optimal when $\rho<$ $\rho^{E}(\beta, \theta, \bar{F}, \pi)$ and $\omega \geq 1$. Combining these two results, we obtain that when $\theta$ is not too large, notifications are optimal if the semi-separating equilibrium $\left(E_{S S}\right)$ does not exist and $\omega \in] \frac{1-\rho}{\rho}, \frac{\rho}{1-\rho}[$. When $\theta$ is close to 1 , it is impossible to guarantee that the lower bound will remain $\frac{1-\rho}{\rho}$. Intuitively, we should obtain that the bound will tend to 1 as $\theta$ tends to 1 : when $\theta$ is close to 1 , ex post control is very ineffective. Therefore, unless it is possible to audit all notifications with probability 1 , the $C A$ stops monitoring ex post and concentrates resources on ex ante control. However, if the $C A$ cannot commit to an audit policy, bad agreements are rather signed than notified. In that case, black list is more efficient than the notification regime. Auditing notifications with probability 1 is possible only when a small number of agreements are notified, and therefore such an equilibrium is similar to the pooling equilibrium $\left(E_{P}\right)$.

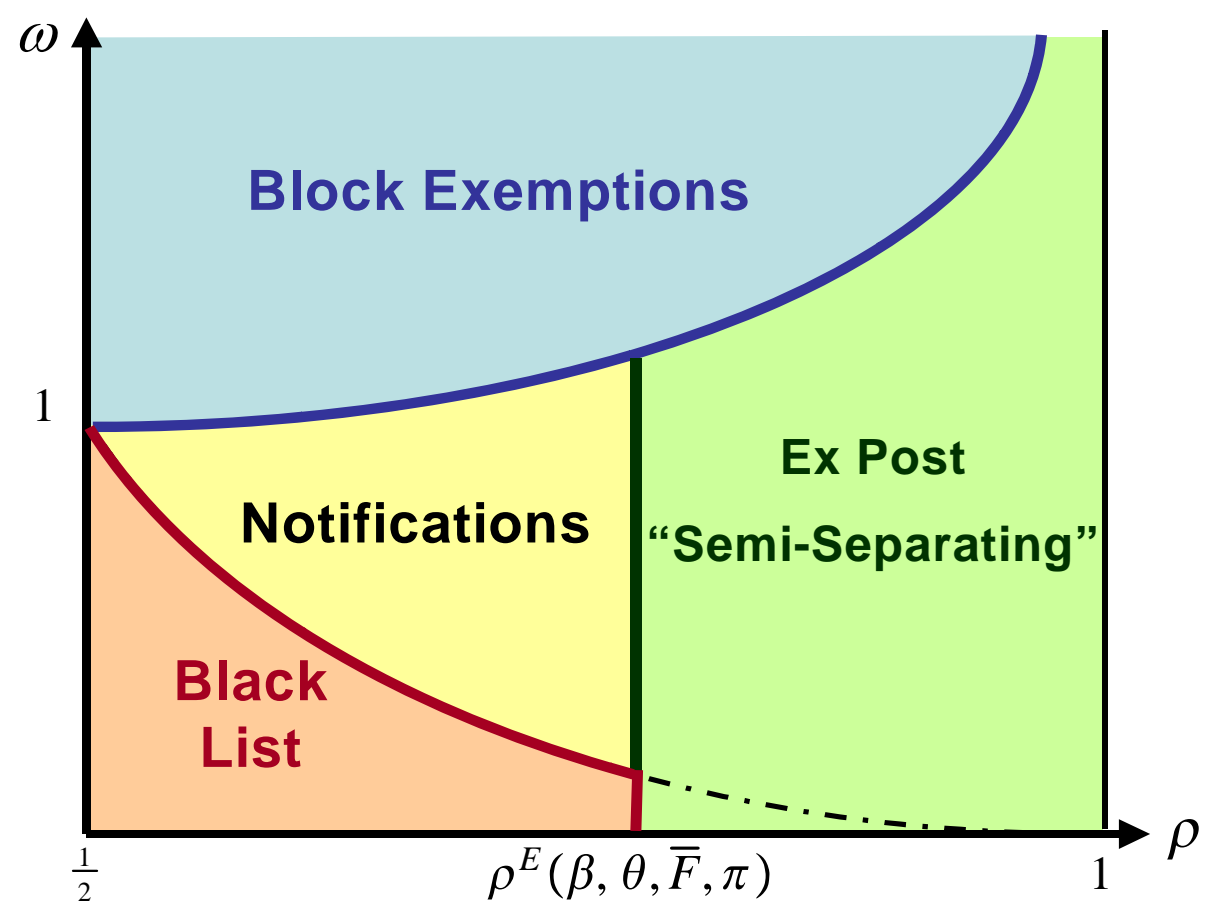

Figure 2: Optimal Audit Regime

Figure 2 summarizes the results of the different propositions. When the signal is accurate enough, a favorable self-selection of the agreements occurs, i.e., some of the bad agreements are not signed. Thus, this is necessarily better than anything possible under a notification regime. The problem with notifications is that it offers a "new safe heaven" for 
firms: because the worst that can happen is to see the agreement being blocked (without fines), it is always worth notifying rather than giving up the chance to implement the agreement. On the other hand, when the signal is not accurate enough, notifications do better than a pure ex post regime for intermediate values of the priors. In the ex post regime, all agreements are signed: it is then preferable to intervene early and this is made possible by the notification system.

\section{Concluding Remarks and Discussion}

We can now interpret our results in the light of the recent reform of European competition policy. As we have shown, the notification regime only dominates when the risk of errors is quite high. This is likely to be the case for a young authority with limited experience like the European Commission was probably in 1962. When the signal's accuracy increases, our analysis suggests to move away from an authorization regime to an exception regime with ex post control. It seems reasonable to assume that after 40 years of experience, the European Commission has acquired this knowledge and that its quality of judgment is now much higher. Note that alternative interpretations of our results are possible: a change of regime could also be explained by affecting the parameters $\beta$ or $\theta$. This would for instance be the case if the $C A$ has become more efficient and now needs less resources to analyse a particular case (keeping the quality of audit constant). The same analysis would hold if we admit that having a better understanding of the markets, the EC is now able to intervene earlier or faster when dealing with a case under the ex post regime.

This paper also allows to analyze the relevance of the two other polar systems. The prior beliefs are then crucial in the decision to use one of these two systems. We show that when the quality of the information collected during an audit (ex ante or ex post) is getting better, the two systems become less useful. Because we have focused on a given category of agreements (i.e., a given value of $\omega$ ), we limit ourselves to using one regime only. It would however be more realistic to assume that the $C A$ faces different categories of agreements (i.e., a distribution of values of $\omega$ ) and has to decide how to treat each category under its budget constraint. Although our model does not allow for that, we can use our results to conjecture what is likely to happen. If the signal is very inaccurate $\left(\rho<\rho^{E}(1, \theta, \bar{F}, \pi)\right)$, the $C A$ has to choose between three regimes: block exemptions, black list and notifications. We should then expect the $C A$ to partition the support of $\omega$ in three intervals: agreements for which $\omega$ is very low (resp. very high) would be banned (resp. legal) per se, whereas those with intermediate values of $\omega$ would be required to 
be notified. If on the other hand, the signal is very accurate $\left(\rho>\rho^{E}(0, \theta, \bar{F}, \pi)\right)$, then a semi-separating equilibrium is always sustainable in the ex post game: we should then expect agreements for which priors are very good (high $\omega$ ) to be considered legal per se, while the other categories would be audited ex post. For intermediate values of $\rho$, the situation is more complex and we could potentially have co-existence of the four regimes.

Finally, let us discuss some issues about the robustness of our results. One of the possible criticism of our model would be that it is usually costly to enter into an agreement and firms therefore incur a non-trivial sunk cost due to the time and resources that it takes to negotiate and structure such an agreement. This would for example mean that firms who only have the opportunity to enter a bad agreement would never do so if the probability of being detected is close to 1 . There are however several reasons why we believe that this is not necessarily true: firstly, the same sunk costs would have to apply in the setting with ex post monitoring, so that the legal exception regime would always be able to induce a more favorable self-selection than the notification regime when the signal is accurate enough. Finally, such sunk costs are likely to be relatively small compared to the benefits that the firms would expect from the agreements so that our results would remain valid. ${ }^{13}$ We believe that this type of argument still holds if we consider filing fees in the notification system as it exists for merger control in the US. This would differ slightly from a sunk cost as it would only be paid in the ex ante monitoring regime. ${ }^{14}$ However, to have any impact these filing fees would have to be substantial. In the U.S., firms have to pay fees that vary from $\$ 45,000$ to $\$ 280,000$ when they notify a merger if the value of the assets under consideration is above $\$ 50$ million: this means that filling fees are relatively small (never more than $0.1 \%$ of these assets). The introduction of filing fees should not change qualitatively our results.

There are also other instruments that the $C A$ might be able to use to increase the efficiency of the different regimes. One such possibility would be to affect the probability of error by setting different standards of proof. To be able to take this into account in our model, we would need to assume that the risk of making type I and II errors are not directly related (in our model we have $\operatorname{Pr}(\operatorname{Err}[I I])=1-\rho=\operatorname{Pr}(\operatorname{Err}[I]))$. If we had different probabilities, say,

$$
\operatorname{Pr}(\sigma=b \mid T=B)=\rho_{B} \text { and } \operatorname{Pr}(\sigma=g \mid T=G)=\rho_{G}
$$

\footnotetext{
${ }^{13}$ Introducing a sunk cost $c$ means considering $\pi_{G}-c$ and $\pi_{B}-c$ instead of $\pi_{G}$ and $\pi_{B}$ respectively. If this cost is small, our analysis remains unchanged.

${ }^{14}$ See for example Besanko and Spulber (1993) who examine the impact of such filing fees on the optimal policy for the case of mergers.
} 
the condition (1) to have a semi-separating equilibrium would write as:

$$
1-\left(\rho_{G}+\pi \rho_{B}\right) \leq \beta(1-\theta) \rho_{B}\left(1-\rho_{G}\right)(1-\pi) .
$$

Therefore by affecting the two probabilities $\rho_{B}$ and $\rho_{G}$, the $C A$ could increase the chances to make a semi-separating equilibrium sustainable. However, once again, when both $\rho_{B}$ are $\rho_{G}$ are close to $\frac{1}{2}$, a semi-separating equilibrium does not exist in the pure ex post control game and notifications will thus remain optimal (at least for intermediate values of the priors $\omega)$.

Finally, another assumption we have made is that the ex post audit is completely random. This might not be the case in practice since some if not most of the ex post audits take place following complaints or revelations about specific markets of firms. This means that we might have underestimated the efficiency of the ex post regime. If this was actually the case, it would mean that pure ex post control if preferable for lower values of $\rho$ but also higher values of $\omega$. However, since not all notifications are analyzed ex ante, we could also believe that the choice of notified agreements that are audited is not random and this would also increase the efficiency of the authorization regime. Overall, it is difficult to know in which direction our results would be modified but once again they would not be qualitatively changed.

- Comment on repeat offenders (Emons?) 


\section{References}

[1] Baron, David and David Besanko (1984), "Regulation, Asymmetric Information and Auditing", Rand Journal of Economics, 15(4), 447-470.

[2] Barros, Pedro Pita (2003), "Looking behind the curtain - effects from modernization of European Competition Policy", European Economic Review, 47(4), 613-624.

[3] Besanko, David and Daniel Spulber (1989), "Antitrust Enforcement Under Asymmetric Information", Economic Journal, 99, 408-425.

[4] Besanko, David and Daniel Spulber (1993), "Contested Mergers and Equilibrium Antitrust Policy", Journal of Law, Economics and Organization, 9(1), 1-29.

[5] Mavroidis, Petros and Damien Neven (2000), "The Modernization of EU Competition Policy : Making the Network Operate", Cahiers de Recherches Economiques 00/17, Université de Lausanne.

[6] Mavroidis, Petros and Damien Neven (2001), "From the White Paper to the Proposal for a Council Regulation. How to Treat the New Kids Around the Block", Legal Issues of Economic Integration.

[7] Motta, Massimo and Michele Polo (2003), "Leniency Programs and Cartel Prosecution", International Journal of Industrial Organization, 21(3), 347-379.

[8] Neven, Damien (2001), "Removing the notification of agreements: some consequences for ex post monitoring", in Meny, von Bogdandy and Mavroidis (eds), Liber Amirocum in Honour of Claus-Dieter Elhermann.

[9] Neven, Damien, Pénélope Papandropoulos and Paul Seabright (1998), Trawling for Minnows: European Competition Policy and Agreements Between Firms, CEPR, London.

[10] Picard, Pierre (1996), "Auditing Claims in Insurance Market with Fraud: The Credibility Issues", Journal of Public Economics, 63, 27-56.

[11] Reinganum, Jennifer and Louis Wilde (1985a), "Income Tax Compliance in a Principal-Agent Framework", Journal of Public Economics, 26, 1-8.

[12] Reinganum, Jennifer and Louis Wilde (1986), "Equilibrium Verification and Reporting Policies in a Model of Tax Compliance", International Economic Review, 27, 739-760. 
[13] Rey, Patrick (2003), "Towards a Theory of Competition Policy", in M. Dewatripont, L. P. Hansen and S. J. Turnovsky (Eds.), Advances in Economics and Econometrics: Theory and Applications (Eight World Congress), Cambridge University Press.

[14] Souam, Saïd (2001), "Optimal Antitrust Policy under Different Regimes of Fines", International Journal of Industrial Organization, 19(1), 1-26.

[15] Spagnolo, Giancarlo (2000), "Optimal Leniency Programs", FEEM Working Paper, \#42.00.

[16] Verouden, Vincent (2003), "Vertical Agreements and Article 81(1) EC: The Evolving Role of Economic Analysis", Antitrust Law Journal, 71(2), 525-575. 


\section{A Preliminary Analysis of the Type $N_{i}(i=1, \ldots, 4)$ Equilibria}

We restrict our attention to the following four types of equilibria:

$N_{1}: \Delta^{*}=((1,0,0),(0,0))$ for $\omega n_{G}^{*} \leq n_{B}^{*} \leq \frac{\rho \omega}{1-\rho} n_{G}^{*}$ and $s_{B}^{*} \geq \frac{\rho \omega}{1-\rho} s_{G}^{*}$.

$N_{2}: \Delta^{*}=((1,0,1),(1,0))$ for $\frac{(1-\rho) \omega}{\rho} n_{G}^{*} \leq n_{B}^{*} \leq \omega n_{G}^{*}$ and $\frac{(1-\rho) \omega}{\rho} s_{G}^{*} \leq s_{B}^{*} \leq \frac{\rho \omega}{1-\rho} s_{G}^{*}$.

$N_{3}: \Delta^{*}=((1,0,0),(1,0))$ for $\omega n_{G}^{*} \leq n_{B}^{*} \leq \frac{\rho \omega}{1-\rho} n_{G}^{*}$ and $\frac{(1-\rho) \omega}{\rho} s_{G}^{*} \leq s_{B}^{*} \leq \frac{\rho \omega}{1-\rho} s_{G}^{*}$.

$N_{4}: \Delta^{*}=((1,0,1),(0,0))$ for $\frac{(1-\rho) \omega}{\rho} n_{G}^{*} \leq n_{B}^{*} \leq \omega n_{G}^{*}$ and $s_{B}^{*} \geq \frac{\rho \omega}{1-\rho} s_{G}^{*}$.

Figure 3 shows when these equilibria might exist.
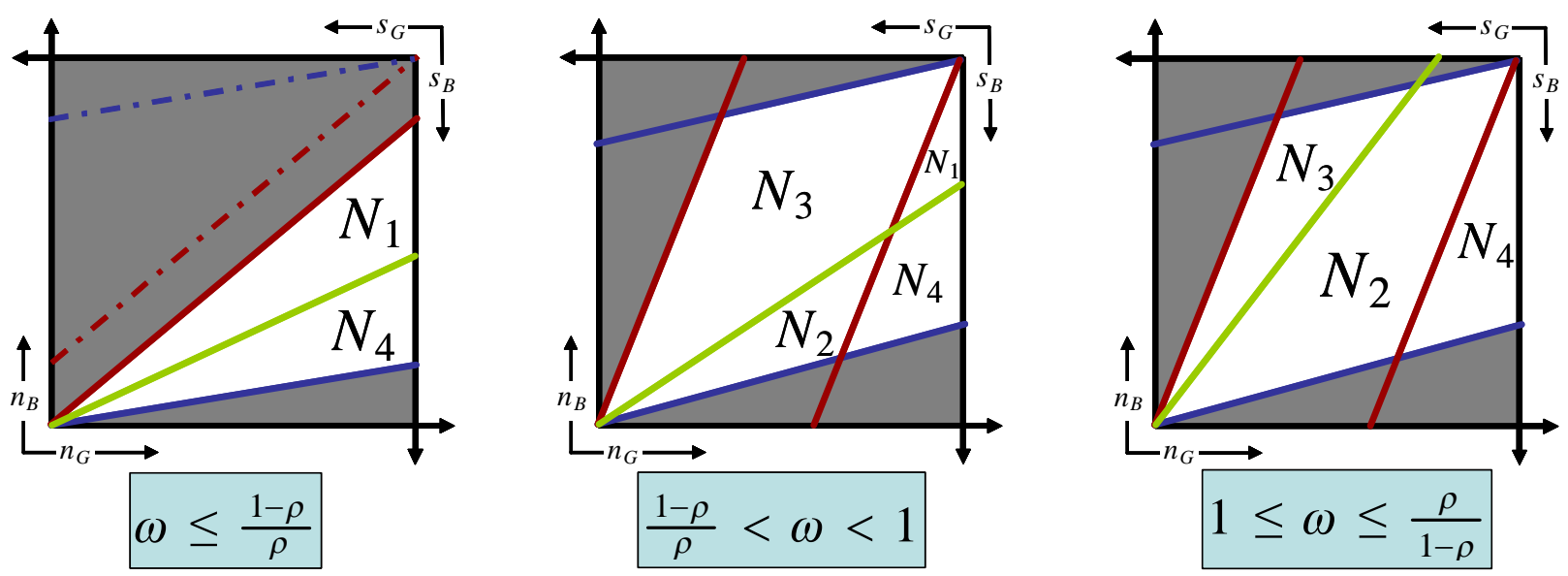

Figure 3: Optimal Decision Profiles

\section{A.1 Type $N_{1}: \Delta^{*}=((1,0,0),(0,0))$}

In this case, the expected welfare is:

$$
\begin{aligned}
W_{1}^{N}= & \left(\rho p_{N} n_{G}+\left(1-(1-\theta) p_{E}\right) s_{G}\right) \omega \\
& -\left((1-\rho) p_{N} n_{B}+\left(1-(1-\theta) p_{E}\right) s_{B}\right) \\
= & \left(\rho \omega n_{G}-(1-\rho) n_{B}\right) p_{N}-\left(s_{B}-\omega s_{G}\right)\left(1-(1-\theta) p_{E}\right) .
\end{aligned}
$$

For such an equilibrium to exist, the fines $F(b)$ and $F(g)$ must be such that the "bad firms" are indifferent between signing and notifying, while the "good firms" must (weakly) 
prefer to notify:

$$
\begin{aligned}
&\left\{\begin{array}{l}
(1-\rho) p_{N}=1-(1-\theta) p_{E}-p_{E}(\rho F(b)+(1-\rho) F(g)) \\
\rho p_{N} \pi \geq\left(1-(1-\theta) p_{E}\right) \pi-p_{E}(\rho F(g)+(1-\rho) F(b))
\end{array}\right. \\
& \Leftrightarrow\left\{\begin{array}{l}
\rho F(b)+(1-\rho) F(g)=\frac{1-(1-\theta) p_{E}-(1-\rho) p_{N}}{p_{E}}, \\
(1-\rho) F(b)+\rho F(g) \geq \frac{\left(1-(1-\theta) p_{E}-\rho p_{N}\right) \pi}{p_{E}} .
\end{array}\right.
\end{aligned}
$$

For these conditions to be satisfied with positive fines, it must therefore be the case that:

$$
1-(1-\theta) p_{E}-(1-\rho) p_{N} \geq 0
$$

\section{A.2 Type $N_{2}: \Delta^{*}=((1,0,1),(1,0))$}

In this case, the expected welfare writes as:

$$
\begin{aligned}
W_{2}^{N}= & \left(\left(1-(1-\rho) p_{N}\right) n_{G}+\left(1-(1-\theta)(1-\rho) p_{E}\right) s_{G}\right) \omega \\
& -\left(\left(1-\rho p_{N}\right) n_{B}+\left(1-(1-\theta) \rho p_{E}\right) s_{B}\right) \\
= & \omega-1+\left(\rho n_{B}-(1-\rho) \omega n_{G}\right)\left(p_{N}-(1-\theta) p_{E}\right)+(\rho-(1-\rho) \omega)(1-\theta) p_{E} .
\end{aligned}
$$

For such an equilibrium to exist, the fine imposed ex post must be such that both types of firms are indifferent between signing and notifying, that is:

$$
\begin{aligned}
& \left\{\begin{array}{l}
1-\rho p_{N}=1-(1-\theta) \rho p_{E}-p_{E} \rho F(b) \\
\left(1-(1-\rho) p_{N}\right) \pi=\left(1-(1-\theta)(1-\rho) p_{E}\right) \pi-p_{E}(1-\rho) F(b)
\end{array}\right. \\
\Leftrightarrow \quad & F(b)=\frac{p_{N}-(1-\theta) p_{E}}{p_{E}}=\frac{p_{N}-(1-\theta) p_{E}}{p_{E}} \pi .
\end{aligned}
$$

Given that $\pi<1$, this imposes to have $\left.\left.p_{N}=(1-\theta) p_{E} \in\right] 0,1-\theta\right]$ and $F(b)=0$. The welfare thus simplifies to:

$$
W_{2}^{N}=\omega-1+(\rho-(1-\rho) \omega)(1-\theta) p_{E} .
$$

\section{A.3 Type $N_{3}: \Delta^{*}=((1,0,0),(1,0))$}

If such an equilibrium exists the expected welfare writes as:

$$
\begin{aligned}
W_{3}^{N}= & \left(\rho p_{N} n_{G}+\left(1-(1-\theta)(1-\rho) p_{E}\right) s_{G}\right) \omega \\
& -\left((1-\rho) p_{N} n_{B}+\left(1-(1-\theta) \rho p_{E}\right) s_{B}\right) \\
= & \omega s_{G}-s_{B}+\left(\rho \omega n_{G}-(1-\rho) n_{B}\right) p_{N}+\left(\rho s_{B}-(1-\rho) \omega s_{G}\right)(1-\theta) p_{E} .
\end{aligned}
$$




\section{A.4 Type $N_{4}: \Delta^{*}=((1,0,1),(0,0))$}

In this case, the expected welfare is equal to:

$$
\begin{aligned}
W_{4}^{N}= & \left(\left(1-(1-\rho) p_{N}\right) n_{G}+\left(1-(1-\theta) p_{E}\right) s_{G}\right) \omega \\
& -\left(\left(1-\rho p_{N}\right) n_{B}+\left(1-(1-\theta) p_{E}\right) s_{B}\right) \\
= & \omega-1+\left(\rho n_{B}-(1-\rho) \omega n_{G}\right) p_{N}+\left(s_{B}-\omega s_{G}\right)(1-\theta) p_{E} .
\end{aligned}
$$

For such an equilibrium to exist, the fines $F(b)$ and $F(g)$ must be such that the "bad firms" are indifferent between signing and notifying, while the "good firms" must (weakly) prefer to notify:

$$
\begin{aligned}
& \left\{\begin{array}{l}
1-\rho p_{N}=1-(1-\theta) p_{E}-p_{E}(\rho F(b)+(1-\rho) F(g)) \\
\left(1-(1-\rho) p_{N}\right) \pi \geq\left(1-(1-\theta) p_{E}\right) \pi-p_{E}(\rho F(g)+(1-\rho) F(b))
\end{array}\right. \\
\Leftrightarrow & \left\{\begin{array}{l}
\rho F(b)+(1-\rho) F(g)=\frac{\rho p_{N}-(1-\theta) p_{E}}{p_{E}} \\
(1-\rho) F(b)+\rho F(g) \geq \frac{\left((1-\rho) p_{N}-(1-\theta) p_{E}\right) \pi}{p_{E}} .
\end{array}\right.
\end{aligned}
$$

Let us first show that there does not exist any equilibrium with $n_{G}<1$. Any such equilibrium would require the two conditions in (6) to be satisfied at equality that is:

$$
\left\{\begin{array}{l}
F(b)=\widetilde{F}(b) \equiv \frac{\left(\rho^{2}-(1-\rho)^{2} \pi\right) p_{N}-(\rho-(1-\rho) \pi)(1-\theta) p_{E}}{(2 \rho-1) p_{E}} \\
F(g)=\widetilde{F}(g) \equiv \frac{-\rho(1-\rho)(1-\pi) p_{N}+(1-(1+\pi) \rho)(1-\theta) p_{E}}{(2 \rho-1) p_{E}} .
\end{array}\right.
$$

In order to have $\widetilde{F}(b) \geq 0$ and $\widetilde{F}(g) \geq 0$, we would need to have:

$$
\frac{\rho-(1-\rho) \pi}{\rho^{2}-(1-\rho)^{2} \pi} \leq \frac{p_{N}}{(1-\theta) p_{E}} \leq \frac{1-(1+\pi) \rho}{\rho(1-\rho)(1-\pi)}
$$

but

$$
\frac{\rho-(1-\rho) \pi}{\rho^{2}-(1-\rho)^{2} \pi} \leq \frac{1-(1+\pi) \rho}{\rho(1-\rho)(1-\pi)} \Leftrightarrow(2 \rho-1)^{2} \pi \leq 0,
$$

which can never be satisfied. Therefore any equilibrium must be such that $n_{G}=1$, and the expected welfare thus simplifies to:

$$
W_{4}^{N}=\omega-1+\left(\rho n_{B}-(1-\rho) \omega\right) p_{N}+\left(1-n_{B}\right)(1-\theta) p_{E} .
$$

\section{B Proof of Proposition 2}

Let us now show that we have (whenever the corresponding equilibria exist),

$$
W_{S S}^{E}=\frac{(2 \rho-1) \omega}{\rho}=\omega-1+\frac{\rho-(1-\rho) \omega}{\rho} \geq \max _{i \in\{1,2,3,4\}} W_{i}^{N} .
$$

For this, let us consider the four types of equilibria $N_{i}$ in turn: 
- $\underline{E_{S S} \text { vs. } N_{1} \text { : }}$

$W_{S S}^{E}-W_{1}^{N}=\frac{(2 \rho-1) \omega}{\rho}-\left(\rho \omega n_{G}-(1-\rho) n_{B}\right) p_{N}+\left(s_{B}-\omega s_{G}\right)\left(1-(1-\theta) p_{E}\right)$.

Using the fact that $p_{N} \leq 1$, this rewrites:

$$
\begin{aligned}
W_{S S}^{E}-W_{1}^{N} & \geq \frac{(2 \rho-1) \omega}{\rho}-\left(\rho \omega n_{G}-(1-\rho) n_{B}\right)+\left(s_{B}-\omega s_{G}\right)\left(1-(1-\theta) p_{E}\right) \\
& \geq \frac{\rho-(1-\rho) \omega}{\rho}-\left(\rho n_{B}-(1-\rho) \omega n_{G}\right)-\left(s_{B}-\omega s_{G}\right)(1-\theta) p_{E} .
\end{aligned}
$$

Rewriting:

$$
\frac{\rho-(1-\rho) \omega}{\rho}=\frac{\rho n_{B}-(1-\rho) \omega n_{G}}{\rho}+\frac{\rho s_{B}-(1-\rho) \omega s_{G}}{\rho}
$$

and using the fact that because $\rho>\frac{1}{2}$ we have:

$$
\frac{(1-\rho)}{\rho}<1 \Rightarrow \frac{\rho s_{B}-(1-\rho) \omega s_{G}}{\rho}>s_{B}-\omega s_{G}
$$

yields:

$$
W_{S S}^{E}-W_{1}^{N}>\frac{\left(\rho n_{B}-(1-\rho) \omega n_{G}\right)(1-\rho)}{\rho}+\left(s_{B}-\omega s_{G}\right)\left(1-(1-\theta) p_{E}\right) \geq 0 .
$$

- $\underline{E_{S S} \text { vs. } N_{2} \text { : }}$

$$
W_{S S}^{E}-W_{2}^{N}=\frac{(\rho-(1-\rho) \omega)\left(1-\rho(1-\theta) p_{E}\right)}{\rho}>0 .
$$

- $\underline{E_{S S} \text { vs. } N_{3} \text { : }}$

$$
\begin{aligned}
W_{S S}^{E}-W_{3}^{N}= & \omega-1+\frac{\rho-(1-\rho) \omega}{\rho}-\omega\left(1-n_{G}\right)+1-n_{B}-\left(\rho \omega n_{G}-(1-\rho) n_{B}\right) p_{N} \\
& -\left(\rho\left(1-n_{B}\right)-(1-\rho) \omega\left(1-n_{G}\right)\right)(1-\theta) p_{E} \\
= & \frac{(\rho-(1-\rho) \omega)\left(1-\rho(1-\theta) p_{E}\right)}{\rho}+\omega n_{G}-n_{B}-\left(\rho \omega n_{G}-(1-\rho) n_{B}\right) p_{N} \\
& +\left(\rho n_{B}-(1-\rho) \omega n_{G}\right)(1-\theta) p_{E} \\
= & \frac{(\rho-(1-\rho) \omega)\left(1-\rho(1-\theta) p_{E}\right)}{\rho}+\left(\rho \omega n_{G}-(1-\rho) n_{B}\right)\left(1-p_{N}\right) \\
& -\left(\rho n_{B}-(1-\rho) \omega n_{G}\right)\left(1-(1-\theta) p_{E}\right) .
\end{aligned}
$$

Using the fact that type $N_{3}$ can only exist if $\rho \omega n_{G} \geq(1-\rho) n_{B}$, this rewrites as:

$\rho\left(W_{S S}^{E}-W_{3}^{N}\right) \geq(\rho-(1-\rho) \omega)\left(1-\rho(1-\theta) p_{E}\right)-\left(\rho n_{B}-(1-\rho) \omega n_{G}\right) \rho\left(1-(1-\theta) p_{E}\right)$. 
Note now that when type $N_{3}$ equilibrium exists, we have:

$$
\rho n_{B}-(1-\rho) \omega n_{G} \leq \rho-(1-\rho) \omega
$$

and combining it with:

$$
\rho\left(1-(1-\theta) p_{E}\right)<1-\rho(1-\theta) p_{E},
$$

we get that $W_{S S}^{E}-W_{3}^{N}>0$.

- $\underline{E_{S S} \text { vS. } N_{4} \text { : }}$

$$
\begin{aligned}
W_{S S}^{E}-W_{4}^{N} & =\frac{\rho-(1-\rho) \omega}{\rho}-\left(\rho n_{B}-(1-\rho) \omega\right) p_{N}-\left(1-n_{B}\right)(1-\theta) p_{E} \\
& =\frac{\left(\rho n_{B}-(1-\rho) \omega n_{G}\right)\left(1-\rho p_{N}\right)}{\rho}+\left(1-n_{B}\right)\left(1-(1-\theta) p_{E}\right) \geq 0 .
\end{aligned}
$$

\section{Proof of Proposition 3}

For $\omega \leq \frac{1-\rho}{\rho}$, we only need to consider the type $N_{1}$ and $N_{4}$ equilibria. Let us take them in turn and check that the expected welfare is never positive.

- Type $N_{1}$ : rewrite the expected welfare $W_{1}^{N}$ as a function of $s_{G}$ and $s_{B}$ :

$$
\begin{aligned}
W_{1}^{N} & =\left(\rho \omega\left(1-s_{G}\right)-(1-\rho)\left(1-s_{B}\right)\right) p_{N}-\left(s_{B}-\omega s_{G}\right)\left(1-(1-\theta) p_{E}\right) \\
& =-(1-\rho-\rho \omega) p_{N}-(2 \rho-1) \omega s_{G} p_{N}-\left(s_{B}-\omega s_{G}\right)\left(1-(1-\rho) p_{N}-(1-\theta) p_{E}\right) .
\end{aligned}
$$

From the preliminary analysis (see Appendix A), we know that for such an equilibrium to exist, it must be the case that $1-(1-\rho) p_{N}-(1-\theta) p_{E} \geq 0$, and thus $W_{1}^{N} \leq 0$.

- $\underline{\text { Type } N_{4} \text { : }}$

$$
\begin{aligned}
W_{4}^{N} & =\omega-1+\left(\rho n_{B}-(1-\rho) \omega\right) p_{N}+\left(1-n_{B}\right)(1-\theta) p_{E} \\
& =\left(1-(1-\rho) p_{N}\right) \omega-\left(1-(1-\theta) p_{E}\right)+\left(\rho p_{N}-(1-\theta) p_{E}\right) n_{B} .
\end{aligned}
$$

From the preliminary analysis (see Appendix A), we know that for such an equilibrium to exist, it must be the case that $\rho p_{N}-(1-\theta) p_{E} \geq 0$. Therefore:

$$
\begin{aligned}
W_{4}^{N} & \leq\left(1-(1-\rho) p_{N}\right) \omega-\left(1-\rho p_{N}\right) \\
& \leq \omega-1+(\rho-(1-\rho) \omega) p_{N} .
\end{aligned}
$$

Given that $p_{N} \leq 1$, this yields:

$$
W_{4}^{N} \leq-(1-\rho-\rho \omega) \leq 0 .
$$




\section{Proof of Lemma 3}

We consider here type $N_{1}$ equilibria for which $n_{G}^{*}=1$ and $n_{B}^{*}=1-2 \varepsilon$ for $\varepsilon>0$ small enough and limit our attention to $\omega \in\left[\frac{1-\rho}{\rho}, 1\right]$. For such an equilibrium to exist, the following conditions must be satisfied:

- The budget allocation $((a, e) \in] 0, \beta\left[^{2}\right.$ with $\left.a+e \leq \beta\right)$ must maximize the expected welfare:

$$
W_{1}^{N}=(\rho \omega-(1-\rho)(1-2 \varepsilon)) p_{N}-2 \varepsilon\left(1-(1-\theta) p_{E}\right)
$$

- Fines imposed ex post must be such that:

$$
\left\{\begin{array}{c}
\rho F(b)+(1-\rho) F(g)=\frac{1-(1-\theta) p_{E}-(1-\rho) p_{N}}{p_{E}}, \\
(1-\rho) F(b)+\rho F(g) \geq \frac{\left(1-(1-\theta) p_{E}-\rho p_{N}\right) \pi}{p_{E}} .
\end{array}\right.
$$

Note that the expected welfare is a strictly increasing function of both $p_{N}$ and $p_{E}$. This implies that in equilibrium the budget must be exhausted, that is $a=\beta-e$. The audit probabilities thus write as:

$$
p_{N}(e)=\frac{\beta-e}{1-\varepsilon}=\text { and } p_{E}(e)=\min \left[1, \frac{e}{\varepsilon}\right] .
$$

The expected welfare function $W_{1}^{N}(e)$ is thus a continuous piecewise-linear function of $e$ and is decreasing on $[\varepsilon, \beta]$. For $e \in[0, \varepsilon[$, we have:

$$
\begin{aligned}
\left.\frac{d W_{1}^{N}}{d e}\right|_{e<\varepsilon} & =-\frac{\rho \omega-(1-\rho)(1-2 \varepsilon)}{1-\varepsilon}+2(1-\theta) \\
& =\frac{2(1-\theta)-(\rho \omega-(1-\rho))-2(2-\theta-\rho) \varepsilon}{1-\varepsilon} .
\end{aligned}
$$

For the expected welfare to be maximized for $e \neq 0$ it must be the case that:

$$
\left.\frac{d W_{1}^{N}}{d e}\right|_{e<\varepsilon} \geq 0 \Leftrightarrow \varepsilon \leq \frac{2(1-\theta)-(\rho \omega-(1-\rho))}{2(2-\theta-\rho)} .
$$

This requires to have:

$$
2(1-\theta)-(\rho \omega-(1-\rho))>0 \Leftrightarrow \omega<\frac{1-\rho+2(1-\theta)}{\rho} .
$$

It remains to determine the minimum fine that is necessary in order for the constraints (7) to be satisfied with positive fines given that in equilibrium, $p_{E}=1$ and $p_{N}=\frac{\beta-\varepsilon}{1-\varepsilon}$. A first condition is to have $\theta \geq(1-\rho) p_{N}$. 
Denote by $\widetilde{F}(b)$ and $\widetilde{F}(g)$ the values that satisfy $(7)$ at equality:

$$
\left\{\begin{array}{l}
\widetilde{F}(b)=\frac{(\rho-(1-\rho) \pi) \theta-\rho(1-\rho)(1-\pi) p_{N}}{2 \rho-1} \\
\widetilde{F}(g)=\frac{(\rho \pi-(1-\rho)) \theta-\left(\rho^{2} \pi-(1-\rho)^{2}\right) p_{N}}{2 \rho-1} .
\end{array}\right.
$$

Therefore:

$$
\begin{aligned}
\widetilde{F}(g) \leq \widetilde{F}(b) & \Leftrightarrow(1-\pi) \theta \geq\left(\rho(1-\rho)(1-\pi)-\left(2 \rho-1-(1-\pi) \rho^{2}\right)\right) p_{N} \\
& \Leftrightarrow(1-\pi) \theta \geq(1-(1+\pi) \rho) p_{N} .
\end{aligned}
$$

If we have $(1-\pi) \theta>(1-(1+\pi) \rho) p_{N}$, then the constraint on the fines writes as $\bar{F} \geq \theta-(1-\rho) p_{N}$ which is always satisfied. When $(1-\pi) \theta \leq(1-(1+\pi) \rho) p_{N}$, the constraint writes as:

$$
\bar{F} \geq \widehat{F} \equiv \frac{(\rho \pi-(1-\rho)) \theta-\left(\rho^{2} \pi-(1-\rho)^{2}\right) p_{N}}{2 \rho-1} .
$$

Note now that:

$$
\widehat{F}=\theta+\frac{\left((1-\rho)^{2}-\rho^{2} \pi\right) p_{N}-(1-\pi) \rho \theta}{2 \rho-1},
$$

and given that $\theta \geq(1-\rho) p_{N}$, we have:

$$
\begin{aligned}
\widehat{F} & \leq \theta+\frac{\left(\left((1-\rho)^{2}-\rho^{2} \pi\right)-(1-\pi) \rho(1-\rho)\right) p_{N}}{2 \rho-1} \\
& \leq \theta-(1-\rho(1-\pi)) p_{N}<\theta .
\end{aligned}
$$

Defining by $\bar{\omega}_{1}(\rho, \theta)=\min \left[1, \frac{1-\rho+2(1-\theta)}{\rho}\right]$, such an equilibrium exists whenever $\theta \geq \beta(1-\rho)$ and $\omega<\bar{\omega}_{1}(\rho, \theta)$. Moreover, we have:

$$
\lim _{\varepsilon \rightarrow 0} W_{1}^{N}=\beta(\rho \omega-(1-\rho))>\max \left[0, W_{P}^{E}\right],
$$

and therefore, for $\varepsilon>0$ small enough, $W_{1}^{N}(\varepsilon)>\max \left[0, W_{P}^{E}\right]$.

\section{E Proof of Lemmas 4 and 5}

For any type $N_{2}$ equilibrium, the expected welfare:

$$
\begin{aligned}
W_{2}^{N} & =\omega-1+\left(\rho n_{B}-(1-\rho) \omega n_{G}\right) p_{N}+\left(\rho s_{B}-(1-\rho) \omega s_{G}\right)(1-\theta) p_{E} \\
& =\omega-1+\left(\rho n_{B}-(1-\rho) \omega n_{G}\right)\left(p_{N}-(1-\theta) p_{E}\right)+(\rho-(1-\rho) \omega)(1-\theta) p_{E},
\end{aligned}
$$

is non-decreasing in both $p_{N}$ and $p_{E}$, and strictly increasing in at least one of them. This implies that, in equilibrium, the available budget must be exhausted: $e=\beta-a$. 
Moreover, $W_{2}^{N}(a)$ is a continuous, piecewise-linear function. We now focus on equilibria for which $\left(W_{2}^{N}\right)^{\prime}=0$ for any $\left.a \in\right] n+\beta-1, n[$. This condition writes as:

$$
\begin{aligned}
& \left(\rho n_{B}-(1-\rho) \omega n_{G}\right)\left(\frac{1}{n}+\frac{1-\theta}{1-n}\right)=\frac{(\rho-(1-\rho) \omega)(1-\theta)}{1-n} \\
\Leftrightarrow & \frac{\rho n_{B}-(1-\rho) \omega n_{G}}{\rho-(1-\rho) \omega}=\frac{(1-\theta) n}{1-\theta n} .
\end{aligned}
$$

Let us now rewrite the condition (8) using the change of notation $y=\frac{n_{B}-n_{G}}{2}$ :

$$
\frac{\rho(y+n)-(1-\rho) \omega(n-y)}{\rho-(1-\rho) \omega}=\frac{(1-\theta) n}{1-\theta n} \Leftrightarrow y=G(n) \equiv-\frac{\rho-(1-\rho) \omega}{\rho+(1-\rho) \omega} \frac{\theta n(1-n)}{1-\theta n} .
$$

Note that the function $G(n)$ is negative, strictly convex in $n$ and such that:

$$
G(0)=G(1)=0, G^{\prime}(0)=-\frac{\rho-(1-\rho) \omega}{\rho+(1-\rho) \omega} \theta \text { and } G^{\prime}(1)=\frac{\rho-(1-\rho) \omega}{\rho+(1-\rho) \omega} \frac{\theta}{1-\theta}
$$

Note now that a type $N_{2}$ equilibrium that satisfies (8) exists if and only if the following conditions are satisfied:

$\left(C_{2-1}\right): \frac{(1-\rho) \omega}{\rho} n_{G}^{*} \leq n_{B}^{*} \leq \omega n_{G}^{*}$ and $\frac{(1-\rho) \omega}{\rho} s_{G}^{*} \leq s_{B}^{*} \leq \frac{\rho \omega}{1-\rho} s_{G}^{*}$.

$\left(C_{2-2}\right)$ : The expected welfare function $W_{2}^{N}(a)$ is maximized for some $\left.a \in\right] 0, \beta[$ such that

$$
p_{N}(a)=(1-\theta) p_{E}(a) \text {. }
$$

\section{E.1 Equilibria with $n^{*}=1-\varepsilon$ (lemma 4)}

Consider an equilibrium for which $n^{*}=1-\varepsilon$, for some $\varepsilon>0$ small enough and focus on values of $\omega>1$. Let us first focus on condition $\left(C_{2-2}\right)$. Given that $n^{*}=1-\varepsilon$, the function $W_{2}^{N}(a)$ is strictly increasing on $[0, \beta-\varepsilon]$ and constant over $[\beta-\varepsilon, \beta]$. Moreover for $a \in[\beta-\varepsilon, \beta]$, we have:

$$
\frac{\beta-\varepsilon}{1-\varepsilon} \leq p_{N} \leq \frac{\beta}{1-\varepsilon} \text { and } 0 \leq(1-\theta) p_{E} \leq 1-\theta,
$$

therefore condition $\left(C_{2-2}\right)$ can be met for some $\varepsilon$ close to 0 if $1-\theta>\beta$. Moreover, solving the condition $p_{N}(a)=(1-\theta) p_{E}(a)$ for $a$ we get:

$$
a=\frac{\beta(1-\theta)(1-\varepsilon)}{\varepsilon+(1-\theta)(1-\varepsilon)} \Rightarrow p_{N}=\frac{\beta(1-\theta)}{\varepsilon+(1-\theta)(1-\varepsilon)}=\beta-O(\varepsilon) .
$$

Let us now rewrite the condition $s_{B} \leq \frac{\rho \omega}{1-\rho} s_{G}$ using the variables $n$ and $y$ :

$$
(1-\rho)(1-n-y) \leq \rho \omega(1-n+y) \Leftrightarrow y \geq-\frac{\rho \omega-1+\rho}{\rho \omega+1-\rho}(1-n) .
$$


Because $G(n)$ is convex, conditions $\left(C_{2-1}\right)$ boil down to:

$$
G^{\prime}(1)<\frac{\rho \omega-1+\rho}{\rho \omega+1-\rho} \Leftrightarrow H(\rho, \omega)>I(\theta),
$$

where:

$$
H(\rho, \omega)=\frac{(\rho \omega-(1-\rho))(\rho+(1-\rho) \omega)}{(\rho \omega+1-\rho)(\rho-(1-\rho) \omega)} \text { and } I(\theta)=\frac{\theta}{1-\theta} .
$$

Now note that $H(\rho, \omega)$ is increasing in $\omega, H(\rho, 1)=1$ and $\lim _{\omega \rightarrow \frac{\rho}{1-\rho}} H(\rho, \omega)=+\infty$, while $I(\theta)$ is increasing in $\theta$ and such that $I(0)=0$ and $\lim _{\theta \rightarrow 1} I(\theta)=+\infty$. Therefore, there exists a threshold $\underline{\omega}_{2}(\rho, \theta) \in\left[1, \frac{\rho}{1-\rho}\left[\right.\right.$ such that for any $\omega>\underline{\omega}_{2}(\rho, \theta)$, condition $(10)$ is satisfied. Moreover, we have that:

$$
\text { for any } \rho, \underline{\omega}_{2}(\rho, \theta)=1 \text { whenever } \theta \leq I^{-1}(1)=\frac{1}{2} \text {. }
$$

Finally, this means that for such a type $N_{2}$ equilibrium the expected welfare writes as:

$$
W_{2}^{N}=\omega-1+\beta(\rho-(1-\rho) \omega)-O(\varepsilon)>\max \left[0, W_{P}^{E}\right] .
$$

\section{E.2 Equilibria with $n^{*}=\varepsilon$ (lemma 5)}

Consider now an equilibrium for which $n^{*}=\varepsilon$, for some $\varepsilon>0$ small enough and focus first on condition $\left(C_{2-2}\right)$. Let us solve the condition $p_{N}(a)=(1-\theta) p_{E}(a)$ for $a$ and check that we have $a \in[0, \varepsilon]$ :

$$
\frac{a}{\varepsilon}=\frac{(1-\theta)(\beta-a)}{1-\varepsilon} \Leftrightarrow a=\frac{\beta(1-\theta) \varepsilon}{1-\theta \varepsilon} \in[0, \varepsilon]
$$

This shows that condition $\left(C_{2-2}\right)$ can always met and in equilibrium we have:

$$
p_{N}=\frac{\beta(1-\theta)}{1-\theta \varepsilon}>\beta(1-\theta),
$$

and therefore:

$$
W_{2}^{N}=\omega-1+\frac{\beta(1-\theta)}{1-\theta \varepsilon}(\rho-(1-\rho) \omega)>W_{P}^{E} .
$$

Let us now rewrite the condition $n_{B} \leq \omega n_{G}$ using the variables $n$ and $y$ :

$$
n+y \leq \omega(n-y) \Leftrightarrow y \geq-\frac{1-\omega}{1+\omega} n .
$$

Because $G(n)$ is convex, conditions $\left(C_{2-1}\right)$ boil down to:

$$
G^{\prime}(0)<-\frac{1-\omega}{1+\omega} \Leftrightarrow J(\rho, \omega) \equiv \frac{(1-\omega)(\rho+(1-\rho) \omega)}{(1+\omega)(\rho-(1-\rho) \omega)}<\theta .
$$


Note first that this condition is always satisfied if $\omega \geq 1$. We now focus on $\omega<1$. Remark that $J(\rho, \omega)$ is decreasing in $\rho$ and $\omega$, and such that:

$$
\left.J(\rho, 1)=0 \text { and } J\left(\rho, \frac{1-\rho}{\rho}\right)=\rho^{2}+(1-\rho)^{2} \in\right] \frac{1}{2}, 1[.
$$

Therefore, there exists a threshold $\widetilde{\omega}(\rho, \theta) \in\left[\frac{1-\rho}{\rho}, 1[\right.$ such that for any $\omega>\widetilde{\omega}(\rho, \theta)$, condition (11) is satisfied.

We finally need to ensure that an equilibrium of this type is preferred to the black list equilibrium:

$$
W_{2}^{N}>0 \Leftrightarrow \omega>\frac{1-\theta \varepsilon-\beta \rho(1-\theta)}{1-\theta \varepsilon-\beta(1-\rho)(1-\theta)}
$$

Note now that:

$$
\lim _{\varepsilon \rightarrow 0}\left(\frac{1-\theta \varepsilon-\beta \rho(1-\theta)}{1-\theta \varepsilon-\beta(1-\rho)(1-\theta)}\right)=\omega_{P}^{E}(\rho, \beta, \theta) .
$$

Therefore, defining:

$$
\widetilde{\omega}_{2}(\rho, \beta, \theta)=\max \left[\widetilde{\omega}(\rho, \theta), \omega_{P}^{E}(\rho, \beta, \theta)\right],
$$

we get that a type $N_{2}$ equilibrium exists and is preferred to both $E_{P}$ and black list whenever $\omega>\widetilde{\omega}_{2}(\rho, \beta, \theta)$.

\section{F Proof of Proposition 4}

From propositions 2 and 3, we already know that the only possibility for the preferred equilibrium to involve notifications is to have:

$$
\rho<\left(\rho<\rho^{E}(\beta, \theta, \bar{F}, \pi)\right) \text { and } \frac{1-\rho}{\rho}<\omega<\frac{\rho}{1-\rho} .
$$

Lemmas 3 and 5 show that there exist equilibria that dominate both the black list and the pooling equilibria of the ex post control game whenever:

$$
\left\{\begin{array}{l}
\text { either } \omega>\max \left[\widetilde{\omega}(\rho, \theta), \omega_{P}^{E}(\rho, \beta, \theta)\right] \\
\text { or } \theta>\beta(1-\rho) \text { and } \omega<\bar{\omega}_{1}(\rho, \theta)
\end{array}\right.
$$

Assuming that $\theta>\frac{\beta}{2}$, we have that $\theta>\beta(1-\rho)$ for any $\rho$. To conclude the proof of the first part of the proposition, it remains to show that $\bar{\omega}_{1}(\rho, \theta)>\widetilde{\omega}(\rho, \theta)$. Note that $\bar{\omega}_{1}(\rho, \theta)$ is a decreasing function of $\rho$ such that:

$$
\bar{\omega}_{1}(1, \theta)=\min [1,2(1-\theta)] \text { and } \bar{\omega}_{1}(\rho, \theta)>\frac{1-\rho}{\rho} .
$$


Moreover, because $J(\rho, \omega)$ is decreasing in both $\rho$ and $\omega, \widetilde{\omega}(\rho, \theta)$ is increasing in $\rho$ whenever it differs from $\frac{1-\rho}{\rho}$. It is therefore enough to check that:

$$
\bar{\omega}_{1}(1, \theta)>\widetilde{\omega}(1, \theta) \Leftrightarrow \min [1,2(1-\theta)]>\frac{1-\theta}{1+\theta},
$$

condition which is necessarily met for any $\theta \in] 0,1[$.

This in particular implies that for any $\frac{1-\rho}{\rho}<\omega<\frac{\rho}{1-\rho}$, there exists an equilibrium involving notifications that is preferred to the pooling equilibrium of the ex post control game. To ensure that one such equilibrium also dominates the black list equilibrium for any $\frac{1-\rho}{\rho}<\omega<\frac{\rho}{1-\rho}$, we have to show that:

$$
\omega_{P}^{E}(\rho, \beta, \theta)<\bar{\omega}_{1}(\rho, \theta)
$$

This is always true if $\bar{\omega}_{1}(\rho, \theta)=1$, and we thus focus on values of $\rho$ for which $\bar{\omega}_{1}(\rho, \theta)<1$. In this case, the condition (12) rewrites as:

$$
\begin{aligned}
& \frac{1-\beta \rho(1-\theta)}{1-\beta(1-\rho)(1-\theta)}<\frac{1-\rho+2(1-\theta)}{\rho} \\
\Leftrightarrow & (2 \rho-1)(1-\beta(1-\theta))<2(1-\theta)(1-\beta(1-\rho)(1-\theta)) \\
\Leftrightarrow \quad & 2(1-\beta(1-\theta)(2-\theta)) \rho<(3-2 \theta)(1-\beta(1-\theta)) .
\end{aligned}
$$

Note first that whenever, $(2+\beta)(1-\theta)>1$, then (13) is satisfied for any $\rho>\frac{1}{2}$. Suppose now that this is not the case (e.g. $\theta$ is too close to 1 and/or $\beta$ too small). In this case, (13) is satisfied whenever:

$$
\rho<\widetilde{\rho}^{N}(\beta, \theta) \equiv \frac{(3-2 \theta)(1-\beta(1-\theta))}{2(1-\beta(1-\theta)(2-\theta))} .
$$

Now compare $\widetilde{\rho}^{N}(\beta, \theta)$ and $\rho^{E}(\beta, \theta, \bar{F}, \pi)$. We can check that $\widetilde{\rho}^{N}(\beta, \theta)$ is decreasing in $\theta$ and such that $\widetilde{\rho}^{N}\left(\beta, \frac{1}{2}\right) \geq 1, \widetilde{\rho}^{N}(\beta, 1)=\frac{1}{2}$. Moreover, $\rho^{E}(\beta, \theta, \bar{F}, \pi)$ is increasing in $\theta$ and such that:

$$
\rho^{E}(\beta, 1, \bar{F}, \pi)=\min \left[\frac{1}{1+\pi}, \frac{1}{\beta \bar{F}}\right]<1
$$

Therefore, there exists a threshold $\left.\bar{\theta}^{N}(\beta, \bar{F}, \pi) \in\right] \frac{1}{2}, 1[$, such that:

$$
\omega_{P}^{E}(\rho, \beta, \theta)<\bar{\omega}_{1}(\rho, \theta) \text {, for any } \theta<\bar{\theta}^{N}(\beta, \bar{F}, \pi) \text {. }
$$

